\title{
Les mouvements des matériaux du lit du Rhône à la suite de la mise en eau de la dérivation de Donzère-Mondragon
}

\section{Movement of bed material in the Rhône after the water was first let into the Donzère-Mondragon diversion channel}

\section{Mard HENRY}

INGÉNIEUR EN GHEF DES PONTS ET CHAUSSÉES DIRECTEUR DE LA COMPAGNE NALIONALE IJU RHONE CHARGÉ DES SERVICES D'ETTUDS
PAR

$\mathrm{ET}$

\author{
Claude GeMaEHIING \\ INGÉNIEUR DES PONTS ET CHAUSSÉES \\ DIRECTEUR-ADJOINT DES RTUDRS \\ A LA COMPAGNE NATIONAIE DU RHONE
}

\section{Mémoire présenté le 28 juin 1957 au Comité technique de la Société Hydrotechnique de France}

\begin{abstract}
Pendant les cinq années qui ont suivi la mise en service de la retenue et de la dérivation de Donzère-Mondragon sur le Rhone, la Compagnie Nationale du Rhóne a procédé à de nombreux: releves des fonds, qui ont permis de preciser qualitativement et quantitativement les conditions de charriage des matérianx dans le lit du fleuve au voisinage de l'aménagement.

L'estimation globale da débit solide de charriage qu'il a été possible d'en tirer est en bon accord avec les résultats donnés par application de la formule de M. Meyer-Peter, compte tena de la dispersion observée dans la granulométrie des matériaux.

Il convient cependant de noter que la saturation des matériaux charriés supposée par cette formule n'est pas toujours réalisée sur le Rhône, en raison de l'etat d'organisation contre l'erosion dans lequel se troube actuellement le lit (seuils rocheux naturels et oubrages de régularisation) et des apports relativement modestes des principaux affluents.

La mise en service sur le Rhône d'aménagements noubeanx et la poursuite des études permettront de compléter ultérieurement ces résultats.
\end{abstract}

\begin{abstract}
During the five years that have followed the bringing into sernice of the reservoir and diversion channel at Donzire-Mondragon on the Rhone, the Compagnie Nationale du Rhone have taken numerous bed samples in order to determine, both quantitatively and qualitatively, the state of material transport in the bed of the river in the zone of the scheme.

The overall estimate of the mass trunsport flow, which it was possible to make from the samples, agrees well with the results obtained by using the formula of $\mathrm{Mr}$. Meyer-Peter, bearing in mind the observed dispersion of the grain sizes of the matenial.

It may be noted, however, that the saturation of the transported materials assumed by this formula is not always realised in the Rhone, on account of the state of organisation against erosion in which the bed now lies, i.e. natural rock sills and regulating structures, coupled with the fact that the main tributaries have only a relatively modest deposit.

The putting into operation of new schemes on the Rhone and the pursuit of investigations will allow these results to be completed eventually.
\end{abstract}

\section{INTRODUCTION DE M. HENRY}

La chute de Donzère-Mondragon est en exploitation depuis cinq ans; pendant ce temps, la Compagnie Nationale du Rhône, qui a construit cet aménagement et exploite les ouvrages concernant l'écoulement des eaux et la navigation, a pu faire diverses observations sur les mouvements du lit du Rhône.
J'ai rédigé à ce sujet, en collaboration avec mon adjoint, M. Gemaehling, une note qui a fait l'objet, le 28 février 1957, d'une communication devant la Section d'Hydraulique Fluviale et Maritime de la S.H.F., et qui est présentée ciaprès par M. Gemaehling.

Pour mettre ce compte rendu dans son cadre, 
il faut se remémorer les préoccupations qui animaient la Compagnie lorsqu'elle procédait aux études de Donzère-Mondragon, au sujet des risques dus aux sables et graviers constituant le lit du fleuve.

Il y a un siècle, avant d'être fixé et régularisé, le lit du Rhône se présentait plutôt mal :

- dans certaines zones, le courant se partageait en plusieurs bras, en se répandant sur une largeur susceptible d'atteindre un kilomètre. Telle était la situation au droit du barrage de Donzère et aussi du barrage supérieur, celui de Rochemaure, qui est en eau depuis juin 1957;

- à chaque confluent il y avait une large zone de divagation, ou bien le Rhône devait péniblement déverser sur le cône de déjection de l'affluent (cas du confluent de la Drôme);

- dans l'ensemble, l'état du fleuve donnait à penser que le lit était extrêmement mobile et que ses variations mettaient en jeu des volumes très importants de gravier.

Dans l'ensemble, l'exécution des travaux de régularisation n'a pas confirmé cette opinion; cependant l'endiguement étroit de Miribel a mis en mouvement des quantités de gravier suffisantes pour que le lit, exhaussé dans la partie aval de l'endiguement, dépasse les digues.

Le premier barrage établi sur le Rhône a été celui de Chèvres, sur le Rhône suisse. Au bout d'une dizaine d'années, on a constaté que la retenue de Chèvres s'engravait dangereusement; MM. les Professeurs Lugeon et Meyer-Peter, consultés, ont conclu à la nécessité d'exécuter des chasses.

Lorsqu'il s'est agi, en 1896, d'aménager la dérivation de Jonage, en amont de Lyon, on n'a pas osé construire un barrage en tête pour obli- ger les eaux à emprunter cette dérivation. Le débit se partageait librement entre le Rhône et la dérivation. Il a fallu attendre $\mathbf{1 9 3 5}$ pour que ce barrage fût construit.

Plus près de nous, l'entretien de la retenue de Chancy-Pougny s'est montré possible grâce à des chasses; on a cependant constaté en aval un abaissement du lit, qui ne parait pas avoir augmenté depuis la mise en eau du barrage de Verbois situé en amont.

Dans l'ensemble, ces constatations incitaient la Compagnie à la prudence.

Le barrage de Donzère étant situé en aval d'une gorge, le défilé de Donzère, il était tentant de choisir un niveau élevé pour la retenue de Donzère, de façon que la dérivation supérieure de Montélimar puisse joindre le Rhône le plus en amont possible, et par exemple, de prendre pour niveau de retenue à Donzère un niveau voisin de celui de la plus forte crue connue.

Par, prudence, le niveau de la retenue a été fixé à 3 mètres au-dessous, ce qui a conduit à faire restituer la chute de Montélimar immédiatement en amont du défilé de Donzère.

Le niveau de retenue étant fixé, le barrage a été équipé de grandes passes, afin de ne pas constituer un obstacle au cours du débit solide. La prise d'eau constituait un objet de préoccupations et a fait l'objet d'études très complètes, d'abord au laboratoire de la Compagnie, puis au laboratoire de Chatou, l'objet essentiel de ces études étant de déterminer des formes telles que ni le gravier, ni le sable charriés par le Rhône ne s'introduisent dans le canal d'amenée.

Enfin, la Compagnie a commandé une drague capable de draguer 100 à $150 \mathrm{~m}^{3} / \mathrm{h}$ à $7 \mathrm{~m}$ de profondeur.

Ces précautions étant rappelées, je laisse à M. Gemaehling le soin de vous donner connaissance des constatations faites depuis la mise en eau.

\section{EXPOSÉ DE M. GEMAEHLING}

Lorsque la Compagnie Nationale du Rhône entreprit, en 1946, l'aménagement du fleuve entre les confluents de l'Isère et de l'Ardèche, on disposait de bien peu de données précises sur le cheminement des matériaux dans le fond du lit. Il était difficile, sinon impossible, de prévoir l'ordre de grandeur du débit solide qui cheminait dans le Rhône et d'arrêter avec toutes certitudes de réussite les précautions à prendre pour éviter l'envahissement de la prise par les galets ou des modifications du lit susceptibles d'entraîner des dommages.
Du point de vue qui nous intéresse ici, le Rhône, à l'instar de certains torrents, semble caractérisé par la forte vitesse de son courant et les dimensions notables des matériaux qu'il transporte. Malgré les travaux de régularisation effectués, le lit est souvent encombré de bancs de gravier, dont l'aspect se modifie après le passage de chaque crue et qui sont découverts en étiage.

Les inquiétudes rappelées par M. Henry, et qui avaient incité à la prudence lors de l'élaboration du projet de Donzère-Mondragon, con- 
duisaient notamment à se poser la question suivante : le Rhône se comporte-t-il comme un fleuve ou comme un torrent?

Pour répondre à cette question, il faut examiner l'allure du lit et la distribution des débits.

\section{A. - Le lit du Rhône en aval de Lyon.}

Sur les $325 \mathrm{~km}$ qui séparent Lyon de la Méditerranée, le Rhône évolue sensiblement sous l'influence des apports importants de ses affluents, tout en conservant certaines caractéristiques qui lui sont propres.

Sans doute, le débit moyen double-t-il entre Lyon et la mer. Mais, à l'inverse de certains de ses affluents ou sous-affluents tels que la Drôme, la Durance ou le Drac, ou d'autres cours d'eau importants tels que la Loire (pour ne citer que quelques rivières à débit solide notable), le Rhône est un fleuve au débit d'étiage relativement important par rapport à son débit moyen et même à ses débits de crue. Qu'il s'agisse du Haut-Rhône (en amont de Lyon) ou du Bas-Rhône (en aval), le rapport entre le débit moyen et le débit d'étiage ne varie guère. Il est compris entre 2,5 et 3,2 , alors que pour la Loire à Orléans, la Drôme et le Drac, ces rapports sont compris entre 14 et 20 .

\section{TABLEAU I}

\begin{tabular}{|c|c|c|c|}
\hline & $\begin{array}{l}\text { DÉBIT } \\
\text { MOYEN } \\
\frac{Q_{m}}{\mathrm{~m}^{3 / \mathrm{s}}}\end{array}$ & $\begin{array}{c}\text { DÉBIT } \\
\text { D'ÉTIAGE } \\
\text { de } 10 \text { jours } \\
\frac{Q \mathrm{e}}{\mathrm{m}^{3} / \mathrm{s}}\end{array}$ & $\begin{array}{l}\text { Rapyont } \\
\frac{Q_{m}}{Q_{e}}\end{array}$ \\
\hline $\begin{array}{l}\text { Haut-Rhône (Génis- } \\
\text { siat) } \ldots \ldots \ldots \ldots \ldots\end{array}$ & 364 & 145 & 2,5 \\
\hline $\begin{array}{c}\text { Bas - Rhône secteur } \\
\text { amont (La Mula- } \\
\text { tière) .......... }\end{array}$ & 1.027 & 325 & 3,15 \\
\hline $\begin{array}{c}\text { Bas - Rhône secteur } \\
\text { central (Le Teil) }\end{array}$ & 1.521 & 560 & 2,7 \\
\hline $\begin{array}{l}\text { Bas - Rhône secteur } \\
\text { aval (Avignon)... }\end{array}$ & 1.600 & 600 & 2,7 \\
\hline Loire (à Orléans) ... & 350 & 25 & 14 \\
\hline
\end{tabular}

Le Rhône est donc alimenté comme l'est un fleuve et non un torrent.'Le travail des débits moyens sur les fonds est d'ailleurs révélé par l'expérience courante: après une grande crue, on constate que certains seuils sont exhaussés d'un mètre et même davantage, mais, en quelques semaines, les débits de 1500 à $2000 \mathrm{~m}^{3} / \mathrm{s}$, qui sont proches des débits moyens, ont ramené les choses à leur état normal.

Sur un torrent, où les eaux moyennes ne durent pas, le même travail est confié aux eaux d'étiage : elles reconstituent bien un lit mineur, mais qui n'a souvent aucun rapport avec l'ancien. C'est ce que nous constatons par exemple sur la Drôme.

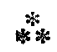

Le Bas-Rhône est divisé en trois secteurs de longueur's à peu près égales, mais aux pentes comme aux débits de charriage différents :

- Le secteur amont, entre les confluents de la Saône et de l'Isère; sa pente moyenne est de $0,50 \mathrm{~m} / \mathrm{km}$; il est peu alimenté en débit solide, parce que le Haut-Rhône ne lui en apporte guère et que la Saône ne charrie que des sables;

- Le secteur central, entre les confluents de l'Isère et de l'Ardèche; sa pente est forte $(0,75 \mathrm{~m} / \mathrm{km})$; il est insuffisamment alimenté en débit solide par ses affluents de rive gauche (Isère, Drôme) et ceux cévenols de rive droite (Eyrieux, Ouvèze), eu égard à sa capacité de transport importante résultant de son débit liquide et de sa pente, de sorte que le lit se creuse à l'amont et s'exhausse à l'aval, le point de basculement se trouvant vers le Teil;

-_ Le secteur aval, au sud du confluent de l'Ardèche, dont la pente décroît graduellement de $0,40 \mathrm{~m} / \mathrm{km}$, pour devenir insignifiante audelà d'Arles. Le débit solide charrié dans ce secteur n'est pratiquement constitué que par les matériaux en provenance de l'amont, du moins jusqu'au confluent de la Durance, et l'on observe qu'il n'arrive à Arles que du sable et de petits graviers.

Le lit naturel du Rhône, tel qu'il existait il y a une centaine d'années, avait été modelé par les possibilités d'érosion et de dépôt. Il ne divaguait pas cependant sur tout le long de son cours : des passages rétrécis naturels, tels que ceux de Vienne ou de Tain, ou artificiels, tels ceux provoqués par les levées d'accès à des ponts, contenaient le lit; des seuils rocheux, comme ceux de Charmes, de l'Homme d'Armés et de BourgSaint-Andéol, s'opposaient à des érosions importantes du lit à leur amont. Cette stabilité du lit, attestée par des monuments très anciens construits en bordure du fleuve, était cependant toute relative. Pour s'en convaincre, il suffit d'examiner une carte où figure la limite des départements riverains du Rhône. Celle-ci coïncidait, sous la Révolution, avec l'axe du lit mineur ou du bras principal du fleuve; elle s'en écarte en de nombreux points à l'heure actuelle. Cette relative stabilité permettait d'assurer, dans des conditions acceptables, l'écoulement général des eaux et le cheminement du débit solide sans 
modifications importantes du lit pouvant mettre en péril des villages lors des crues, mais elle rendait très précaire la navigation, qui a besoin d'un chenal continu, au tirant d'eau suffisant en étiage. Il y a à peine un siècle, il existait entre Lyon et la mer plus de 150 passages où le tirant d'eau était, en étiage, inférieur à $1,60 \mathrm{~m}$, et dont certains, tels le confluent de la Drôme, n'avaient que $0,50 \mathrm{~m}$.

L'aménagement du fleuve à courant libre entre Lyon et la mer, essentiellement réalisé entre 1880 et 1900 par le Service Spécial du Rhône, notamment sous l'impulsion de Girardon, a permis de fixer le lit et de créer un chenal navigable stable au tirant d'eau de $1,60 \mathrm{~m}$ en étiage. Le succès de ces travaux de correction a été complet, alors que l'on connaît les inconvénients qui sont résultés de la rectification entreprise au dix-neuvième siècle de certains autres grands cours d'eau européens.

Sur le Rhône, on a procédé de manière très prudente : le lit mineur, dont on a maintenu sensiblement la longueur et la pente naturelle, a été entièrement fixé et recalibré de manière à assurer à la navigation un mouillage suffisant; les bras morts ou lônes, où le Rhône avait tendance à divaguer, ont été partiellement obturés à leur extrémité amont de manière à concentrer les eaux basses et moyennes dans le lit mineur tout en permettant le passage des débits de crue. Les berges ont été renforcées par des digues longitudinales submersibles et par des épis, les digues étant, s'il y a lieu, réunies à la terre ferme par des tenons transversaux entre lesquels le colmatage peut s'effectuer. Grâce à ces travaux, le lit mineur s'est complètement stabilisé et les fonds se conservent naturellement sans aucun travail d'entretien ou de dragage.

Les principes qui ont été adoptés pour régulariser le Bas-Rhône ont limité, dans toute la mesure du possible, les inconvénients qu'aurait procuré un endiguement insubmersible et étroit. On peut même se demander si, dans l'ensemble, il y a eu réduction de la pente : il semble cependant que le lit ait légèrement basculé autour d'un point situé vers le Teil, le niveau de l'étiage s'étant abaissé en moins d'un siècle de $0,95 \mathrm{~m}$ à Valence et exhaussé de $1,10 \mathrm{~m}$ à Pont-SaintEsprit. Mais ce basculement est resté suffisamment léger, au point qu'aucune conséquence appréciable n'a pu être rèlevée ni sur les niveaux de nappe, ni sur ceux des crues. Il n'est d'ailleurs apparu aucun nouveau rapide sur les seuils rocheux existant en amont du point de basculement.

La Saône n'apporte que du sable et le lit du Rhòne s'est creusé à son confluent de $0,30 \mathrm{~m}$ en cinquante années. Jusqu'à Tain, le Rhône, qui ne reçoit pas d'affluent important, a un débit solide modéré et ses fonds sont stables.
De Tain à la Dròme, l'abaissement a, par contre, été sensible et il est remarquable que ce secteur encadre le confluent de l'Isère, au droit duquel le creusement a été maximum. Cela s'explique par le fait que l'Isère, qui ne charrie guère de matériaux dans son secteur aval, en apporte fort peu au Rhône à l'heure actuelle. Le lit de cette rivière s'exhausse, particulièrement dans la région de Moirans, et les retenues des aménagements hydroélectriques de la BasseIsère (Beaumont-Monteux, Pizancon et La Vanelle) ne reçoivent pas beaucoup de graviers.

La Drôme n'apporte qu'une quantité modérée de graviers au Rhône. Son lit inférieur s'exhausse. Tout au plus remarque-t-on que, sur $3 \mathrm{~km}$ en aval du confluent, les pentes du Rhône sont très variables (de 0,50 à $1,50 \mathrm{~m} / \mathrm{km}$ ), ce qui paraît dénoter, sur cette zone de « digestion » des gravier's apportés par la Drôme, la présence de dunes qui sont peu à peu poussées vers l'aval et écrêtées par le courant du fleuve.

Vers Pont-Saint-Esprit, le lit du Rhòne s'exhausse peut-être parce qu'il s'agit du basculement dont nous avons parlé, peut-être également parce que le Rhône ne parvient pas à entraîner les apports de l'Ardeche, aux crues particulièrement violentes. Peu en amont de la restitution du canal de Donzère-Mondragon, il existe notamment une zone assez instable, la boucle de Lamiat, où des incidents compromeltant la stabilité des berges se sont produits lors d'une grande crue en 1935.

Enfin, le lit du fleuve dans le secteur aval du Bas-Rhône semble s'exhausser lentement. Des graviers parviennent jusqu’à Soujean, situé en amont d'Arles, où une entreprise de dragages s'est installée. Les graviers ne paraissent pas cheminer au-delà, grâce aux dispositions prises en 1937 à la suile d'essais sur modèle.

D'une façon générale, le lit du Rhône est constitué par des alluvions grossières. Pour les alluvions constituant le fond du lit dans la région de Donzère, on a trouvé des zones de grande étendue où les graviers de dimensions supérieures à $100 \mathrm{~mm}$ représentent $25 \%$ du volume, et ceux inférieurs à $25 \mathrm{~mm}, 35 \%$. Les analyses granulométriques des graviers correspondant aux remblaiements de la retenue montrent que l'on a presqu'en permanence $30 \%$ de matériaux supérieurs à $80 \mathrm{~mm}$, ce qui est légèrement inférieur au pourcentage des alluvions en place depuis longtemps. Il semble donc que les matériaux constituant le fond $d u$ lit aient une granulométrie plus grosse que ceux situés à une certaine profondeur, en raison du balayage dû aux crues moyennes. D'ailleurs dans les études sur modèle réduit, la rugosité du lit et la fidélité avec les lignes d'eau naturelles ne sont obtenues qu'au moyen de matériaux desquels on a retiré la majorité des éléments les plus fins. 
En maintes régions, le lit du Rhône est rocheux, parfois sur des distances assez longues, ou pavé naturellement par de gros matériaux. Les fonds rocheux sont soit à nu, comme à l'Homme d'Armes et à Bourg-Saint-Andéol, soit recouverts d'une épaisseur de graviers ne dépassant guère un mètre, comme à Saint-Etiennedes-Sorts. Ces fonds stables, dont aucun n'a été transformé en rapide par la régularisation, contribuent efficacement à fixer le lit à leur amont.

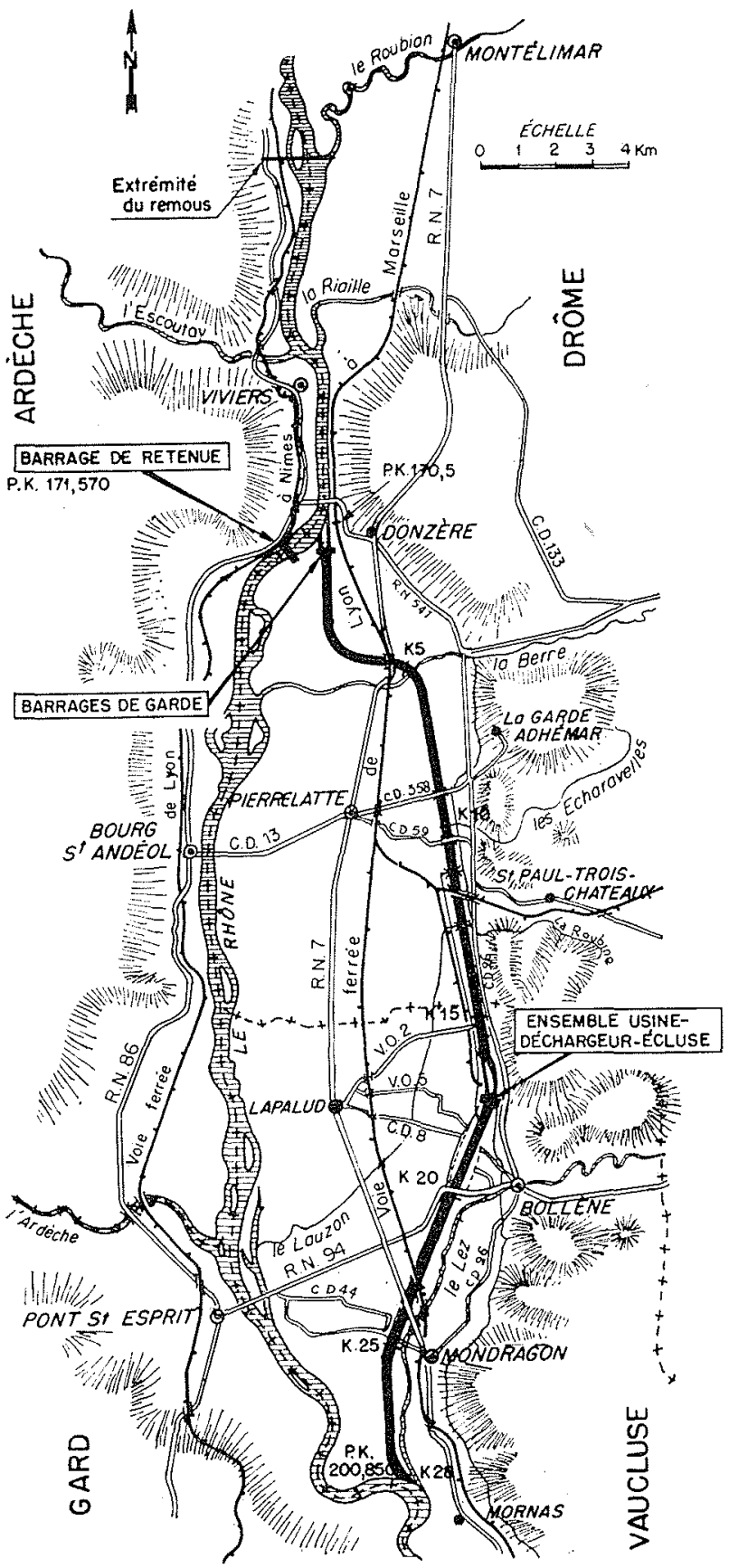

Fig. 1. - Plan d'ensemble de l'aménagement de Donzère-Mondragon.

\section{B. - Influence théorique de l'aménagement de Donzère-Mondragon sur le chemine- ment du débit solide.}

L'aménagement de Donzère-Mondragon (fig. 1) comporte essentiellement un canal de dérivation de $28 \mathrm{~km}$ de longueur, court-circuitant $31 \mathrm{~km}$ de Rhône, et un barrage de retenue situé à $1 \mathrm{~km}$ en aval de l'entrée de la dérivation. Au point kilométrique 17 du canal, se trouvent l'usine hydroélectrique André Blondel, d'une puissance installée de $300000 \mathrm{kVA}$ et une écluse permettant à la navigation de franchir la chute, qui atteint $26 \mathrm{~m}$ environ en étiage.

Rappelons que le débit d'étiage ( 10 jours par an) du Rhône est de l'ordre de $560 \mathrm{~m}^{3} / \mathrm{s}$, soit le

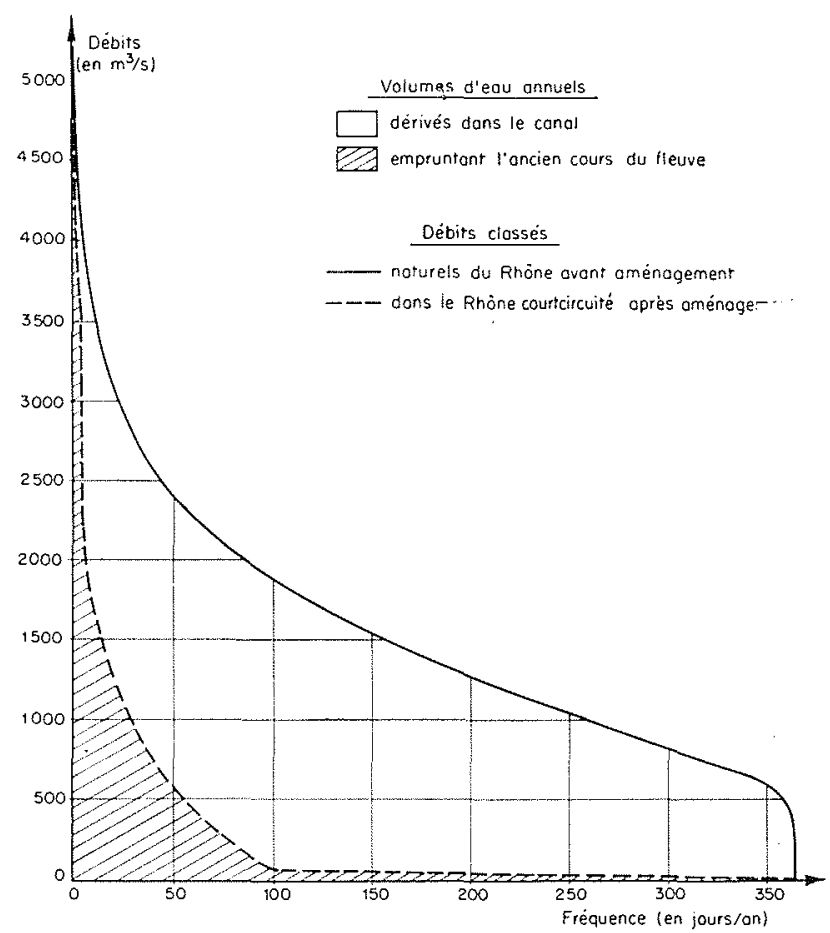

Fra. 2. - Courbe des débits classés du Rhône à Joviac (années 1911 à 1949).

tiers environ du débit moyen $\left(1630 \mathrm{~m}^{3} / \mathrm{s}\right)$, lequel n'est dépassé que 5 mois par an (fig. 2). La crue annuelle dépasse $4100 \mathrm{~m}^{3} / \mathrm{s}$ et la crue millénaire est estimée à $10000 \mathrm{~m}^{3} / \mathrm{s}$. Le débit dérivé dans le canal est de $1530 \mathrm{~m}^{3} / \mathrm{s}$ minimum. Pendant 7 mois par an, le Rhône court-circuité ne recevra donc que le débit réservé, qui a été fixé à $60 \mathrm{~m}^{3} / \mathrm{s}$, soit environ le $1 / 10^{\mathrm{e}} \mathrm{du}$ débit d'étiage du fleuve. Le reste du temps, c'est-à-dire en période d'eaux moyennes ou hautes, le Rhône court-circuité verra son débit amputé de plus de $1.500 \mathrm{~m}^{3} / \mathrm{s}$ par rapport au débit naturel du fleuve.

Le niveau normal de la retenue, mesuré au droit de la prise, est (58); ce niveau, qui dépasse 
de $4,85 \mathrm{~m}$ le niveau d'étiage naturel, est le niveau sous lequel s'écoule la crue annuelle. Le niveau de la plus haute crue connue se situe à $2,50 \mathrm{~m}$ au-dessus du niveau de la retenue. La retenue de Donzère est donc une retenue basse, pour laquelle il est possible de retrouver les conditions d'écoulement naturelles au cours de crues non exceptionnelles.

La retenue a, en étiage, une longueur de $8,5 \mathrm{~km}$. Pour le débit d'une crue modérée ( $\left.3000 \mathrm{~m}^{3} / \mathrm{s}\right)$ mais fréquente, le remous s'étend seulement sur $4,5 \mathrm{~km}$ de longueur et l'effet de retenue n'est vraiment sensible que sur une fraction de cette distance. Pour des crues de l'ordre de $5000 \mathrm{~m}^{3} / \mathrm{s}$, il n'y a plus guère d'effet de retenue et la dérivation d'un débit appréciable dans le canal provoque même un abaissement des lignes d'eau sur une certaine distance en amont de la prise.

La prise d'eau a fait l'objet de longues et minutieuses études sur modèle réduit au laboratoire de la Compagnie Nationale du Rhône, puis au Laboratoire National d'Hydraulique de

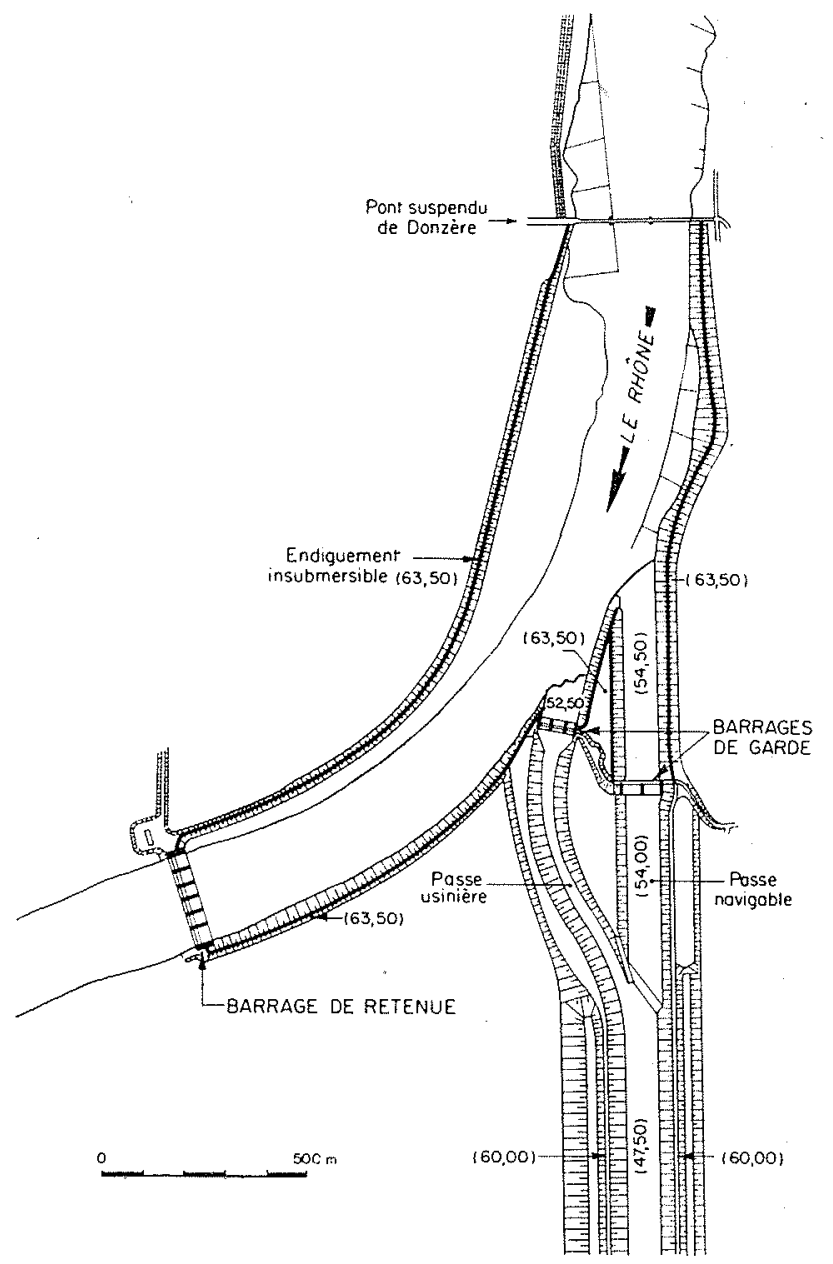

Fig. 3. - Prise d'eau de la dérivation de Donzère-Mondragon.
Chatou, dont notre directeur général, M. Delattre, a rendu compte au cours d'un exposé fait en 1953 devant la S.H.F. (fig. 3).

L'expérience acquise au cours de cinq années d'exploitation a pleinement confirmé les prévisions déduites du comportement du modèle réduit, ce qui fait honneur au Laboratoire de Chatou, qui a eu la lourde tâche de fixer les formes définitives de l'entrée.

Alors que l'on a dérivé en moyenne dans le canal plus des trois quarts du débit liquide du fleuve, on peut affirmer qu'il n'est pas entré de débit solide de charriage dans la dérivation, tandis qu'un volume de matériaux supérieur au million de $\mathrm{m}^{3}$ a transité à travers toute la retenue et franchi le barrage.

Les considérations qui précèdent permettent de supputer le sens des évolutions du lit du Rhône dans les divers secteurs intéressés par l'aménagement de Donzère-Mondragon.

Dans la retenue, qui est basse, l'effet de surélévation du plan d'eau dû à la présence du barrage diminue notablement quand le débit croît et il est pratiquement annulé pour les fortes crues. On peut donc s'attendre à un engravement modéré de la retenue, peu en amont de la prise, dû au relèvement des lignes d'eau pour les seules crues moyennes, et à un engravement assez sérieux entre la prise et le barrage pour toutes les crues, dù à la ponction de débit dans la dérivation.

Le Rhône court-circuité ne reçoit plus qu'une faible part de débit du fleuve et sa courbe de débits classés devient analogue à celle d'un torrent, ainsi que nous l'avons vu sur le graphique précédent. Les débits de l'ordre de $2000 \mathrm{~m}^{3} / \mathrm{s}$, qui dans le Rhône libre rabotent les seuils, lentement, mais avec efficacité, n'existent plus que pendant quelques jours par an; c'est un élément de stabilité qui disparaît. En outre, il est à craindre que le Rhône court-circuité ne puisse acheminer tout le débit solide qui franchit le barrage; aussi est-il à présumer que des dépôts se formeront immédiatement à l'aval de celui-ci.

A l'approche de la restitution, la vitesse des eaux diminue, du fait du remous d'exhaussement imputable aul débit dérivé; il y a risque d'engravement dans cette zone, qui était d'ailleurs instable dans la situation naturelle.

Enfin, en aval de la restitution, les eaux du fleuve non saturées en gravier, grâce à l'appoint des eaux claires du canal, auront tendance à éroder les fonds.

L'observation régulière de l'évolution du lit, dans tout le tronçon du Rhône intéressé par l'aménagement a permis de constater dans quelle mesure s'étaient produites les évolutions auxquelles on pouvait s'attendre. 


\section{C. - Les débits du Rhône depuis la mise en eau de l'aménagement.}

Depuis la mise en eau de l'aménagement de Donzère-Mondragon en juillet 1952 jusqu'en juin 1957, soit en cinq années, onze crues du Rhône, d'importances diverses, se sont produites, étant entendu que nous appelons crue toute montée des eaux au-delà d'un débit de $2500 \mathrm{~m}^{3} / \mathrm{s}$ correspondant à un charriage appréciable.

Ces crues sont les suivantes, en les classant d'après leur débit moyen journalier maximum :

Trois crues inférienres à $3000 \mathrm{~m}^{3} / \mathrm{s}$ en avril $1954\left(2500 \mathrm{~m}^{3} / \mathrm{s}\right)$, en juin $1953(2700$ $\left.\mathrm{m}^{3} / \mathrm{s}\right)$ et en janvier 1956 (2850 $\left.\mathrm{m}^{3} / \mathrm{s}\right)$;

Deux crues comprises entre $3000 \mathrm{~m}^{3} / \mathrm{s}$ et $4000 \mathrm{~m}^{3} / \mathrm{s}$, en juin $1955\left(3200 \mathrm{~m}^{3} / \mathrm{s}\right)$ et en août $1954\left(3400 \mathrm{~m}^{3} / \mathrm{s}\right)$;

Deux crues comprises entre $4000 \mathrm{~m}^{3} / \mathrm{s}$ et $5000 \mathrm{~m}^{3} / \mathrm{s}$, la premièrè décembre 1953 (une seule journée à $4100 \mathrm{~m}^{3} / \mathrm{s}$ ), mais avec des débils de l'ordre de $3000 \mathrm{~m}^{3} / \mathrm{s}$ soutenus pendant près d'un mois, la seconde en septembre $1956\left(4600 \mathrm{~m}^{3} / \mathrm{s}\right)$;

- Quatre crues supérieures à $5000 \mathrm{~m}^{3} / \mathrm{s}$, dont trois successives en décembre 1954 (5 400 $\left.\mathrm{m}^{3} / \mathrm{s}\right)$, en janvier $1955\left(6030 \mathrm{~m}^{3} / \mathrm{s}\right.$, la plus forte depuis la mise en eau) et en février $1955\left(5300 \mathrm{~m}^{3} / \mathrm{s}\right)$ et la quatrième en février $1957\left(5800 \mathrm{~m}^{3} / \mathrm{s}\right)$. Il est à noter qu'il est exceptionnel d'observer trois crues supérieures à $5000 \mathrm{~m}^{3} / \mathrm{s}$ se succédant sans interruption; le débit est resté supérieur à $2000 \mathrm{~m}^{3} / \mathrm{s}$ pendant près de trois mois, supérieur à $4000 \mathrm{~m}^{3} / \mathrm{s}$ pendant plus de trois semaines et supérieur à $5000 \mathrm{~m}^{3} / \mathrm{s}$ pendant 16 jours.

Au total, pendant les cinq années d'observations, les durées pendant lesquelles le débit est resté supérieur à une valeur donnée sont les suivantes :

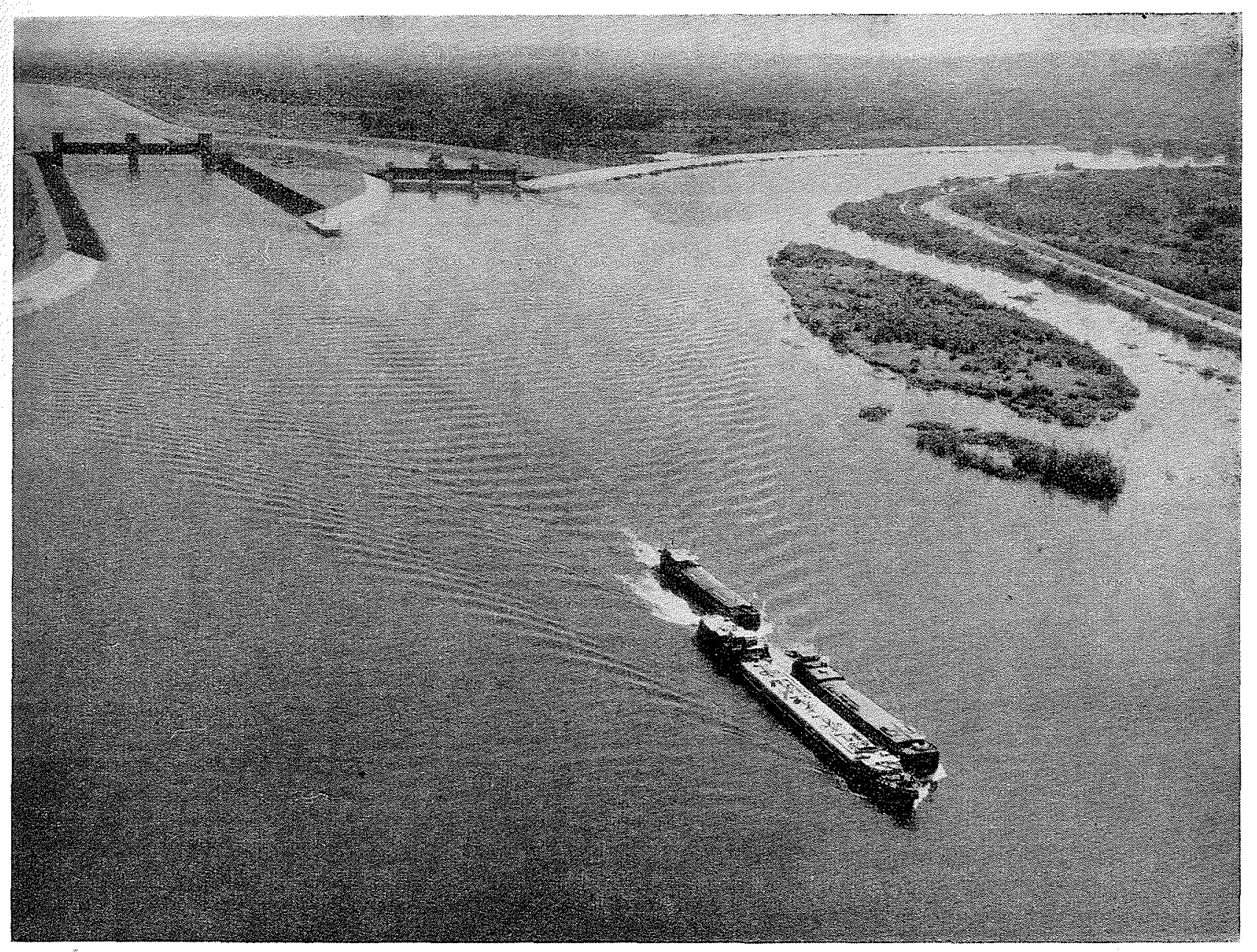

Fig. 4. - L'entrée de la dérivation vue du déflé de Donzère. 


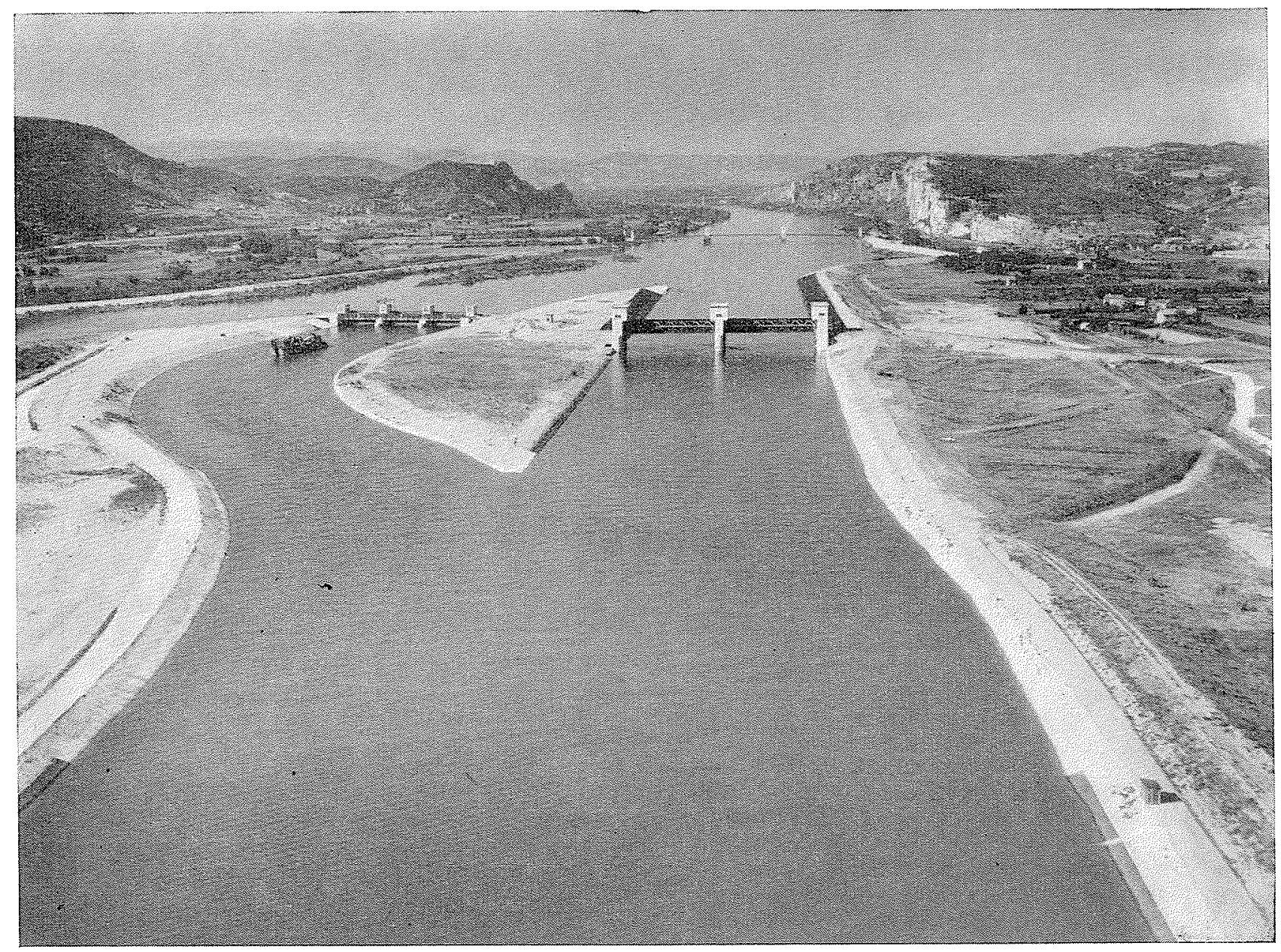

FIG. 5. - L'entrée de la dérivation vue du canal.

Photo Gellard.

- 321 jours, soit près d'une année, au-dessus de $2000 \mathrm{~m}^{3} / \mathrm{s}$;

- 95 jours, soit 3 mois environ, au-dessus de $3000 \mathrm{~m}^{3} / \mathrm{s}$;

- 33 jours, soit 1 mois environ, au-dessus de $4000 \mathrm{~m}^{3} / \mathrm{s}$;

- 19 jours, au-dessus de $5000 \mathrm{~m}^{3} / \mathrm{s}$;

- 2 jours, au-dessus de $6000 \mathrm{~m}^{3} / \mathrm{s}$.

\section{D. - Moyens d'observation des fonds.}

Les moyens d'investigation auxquels nous avons eu recours pour suivre les variations $d u$ lit, ont consisté à relever des profils en travers tous les $100 \mathrm{~m}$, soit par le procédé classique de la perche pour le Rhône court-circuité où règnent de faibles profondeurs et de faibles vitesses de courant, soit par l'utilisation d'un sondeur à ultra-sons dont est équipée une vedette, pour le restant de la longueur du fleuve intéres- sée par l'aménagement. Dans la retenue, les profils ont été levés après chaque crue; dans le Rhône court-circuité, ils l'ont été après les fortes crues et les chasses. En outre, de nombreux relevés de lignes d'eau et des jaugeages ont été effectués.

Le relevé des profils au moyen d'une perche est très facile et précis. "On peut admettre qu'il n'y" a pas d'erreur systématique dans les mesures et que les erreurs de cubatures se compensent. Il n'y a pas de correction à apporter aux chiffres bruts des engravements et des dégravements.

En ce qui concerne les relevés au moyen de l'appareil à ultra-sons, le constructeur garantit une précision de $10 \mathrm{~cm}$. Comme la retenue a $8 \mathrm{~km}$ de longueur et $200 \mathrm{~m}$ de largeur, soit environ 1,5 million de $\mathrm{m}^{2}$, cette erreur de $10 \mathrm{~cm}$ sur les hauteurs, quoique faible, conduirait, si elle était systématique, à une connaissance des volumes de matériaux à $150.000 \mathrm{~m}^{3}$ près. Il s'agirait donc d'une erreur notable, d'un ordre de 


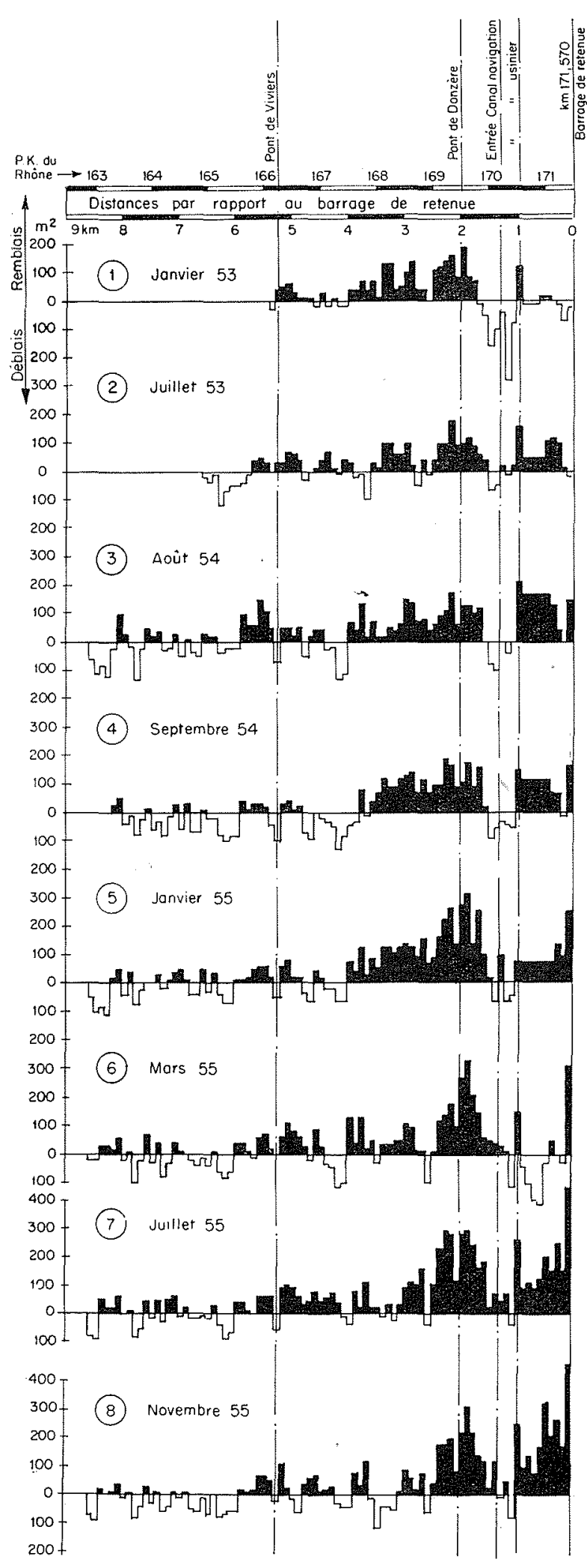

- Par profil -

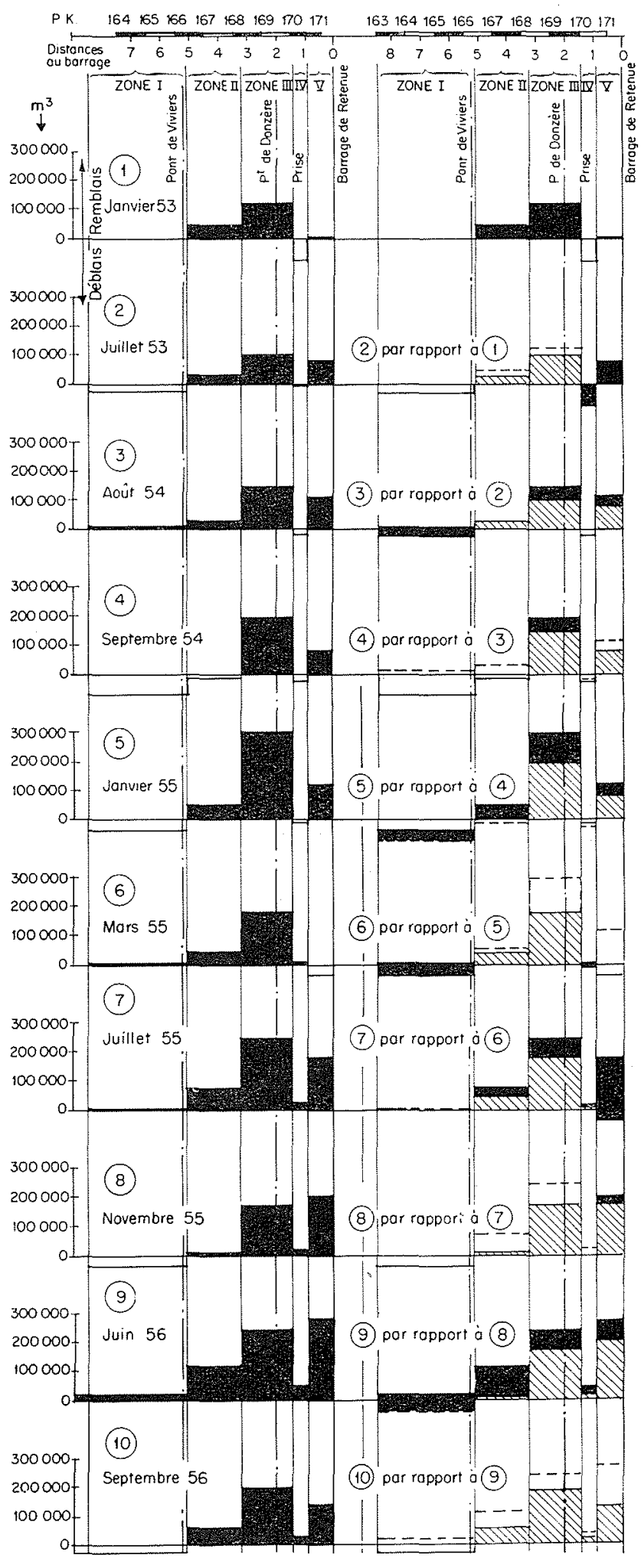

- Par zone -

FIG. 6 et 7. - Evolutions successives des fonds de la retenue 
grandeur analogue au volume des dépôts ou des déblais qui peuvent se manifester entre deux relevés successifs.

Nous avons pu réduire sensiblement cette erreur par une série de moyens sur lesquels je ne m'étendrai pas. Ils ont consisté soit en de nombreux contrôles effectués à la perche, soit en des sondages répétés de zones connues oì les fonds n'évoluaient pas, de manière à réétalonner l'appareil en cours d'opération et à déceler une légère dérive éventuelle du zéro de l'appareil. Ces opérations ont permis de déterminer de quelles corrections il fallait affecter les chiffres bruts obtenus.

\section{E. - Evolution des fonds de la retenue.}

Après chaque crue, il a été procédé à un relevé complet des fonds de la retenue, sauf entre les crues de janvier et février 1955 qui se sont succédées. Nous disposons ainsi, outre le levé initial, de dix relevés des fonds auxquels s'ajoutent deux relevés supplémentaires, effectués à titre de contrôle des résultats, pour pouvoir apprécier le degré de précision des mesures.

A partir de ces renseignements, nous avons établi divers graphiques et plans de manière à nous rendre compte des évolutions du lit :

- Un premier graphique représente, à la fin de chaque crue, les sections de remblais et de déblais, profil par profil (fig. 6).

Ce graphique a mis en évidence un certain nombre de profils successifs qui se comportaient de la même façon et pouvaient être groupés pour constituer une zone au comportement bien défini. Il a en outre été observé que ces zones étaient séparées entre elles par un ou deux profils qui ne se modifiaient pas, et l'on a remarqué que ces profils stables correspondaient aux sections dans lesquelles la vitesse moyenne était la plus faible avant la mise en service de l'aménagement. Ces sections, qui se placent à des sommets de courbes (mouilles), se révèlent particulièrement résistantes à l'engravement.

- Un second graphique représente les volumes de remblais ou de déblais dans les cinq zones que le graphique précédent a permis de mettre en évidence (fig. 7).

Ce graphique permet de préciser le comportement de chaque zone qui est le suivant :

UnE zone $\mathrm{N}^{\circ} 1$, depuis l'amont de la retenue jusque vers le pont de Viviers où les variations du lit sont insignifiantes. Dans cette zone, l'effet de retenue est très faible ou même inexistant pour les débits de charriage, ce qui explique que la mise en service de l'aménagement n'ait eu aucune répercussion sur les fonds;

UNE ZONE $\mathrm{N}^{\circ} 2$, allant du pont de Viviers jusqu'à un point situé à peu près à mi-distance entre les ponts de Viviers et de Donzère.

C'est la zone de commencement des dépòts, et ceux-ci deviennent rapidement appréciables lorsqu'on se déplace de l'amont vers l'aval, étant cependant noté que les mouilles, sections où la vitesse moyenne était la plus faible avant aménagement, montrent une remarquable résistance à l'engravement, qui est limité aux seuils. Comme il s'agit des dépôts situés le plus en amont, la drague d'entretien de la retenue que la Compagnie Nationale du Rhône avait fait construire pour parer à toute éventualité, est venue y travailler. Les dragages, qui ont porté sur $200000 \mathrm{~m}^{3}$, et la chasse de septembre 1956 ont eu pour effet de limiter les engravements autour de $40000 \mathrm{~m}^{3}$, soit une réduction de section de $20 \mathrm{~m}^{2}$ seulement pour des profils représentant une section mouillée de l'ordre de $2500 \mathrm{~m}^{2}$ pour le débit moyen de $1500 \mathrm{~m}^{3} / \mathrm{s}$;

Une zONE $\mathrm{N}^{\circ} 3$, allant de la précédente à l'entrée double. C'est une zone où les dépôts se sont manifestés rapidement. En 1954, l'engravement avait atteint $200000 \mathrm{~m}^{3}$. Depuis cette époque, iI reste voisin de $250000 \mathrm{~m}^{3}$ et l'ouverture du barrage lors des grandes crues ou des chasses provoque la régression partielle des remblais;

UNE ZONE $\mathrm{N}^{\circ} 4$, correspondant à l'entrée double, qui est restée remarquablement stable. Les seuils des entrées fonctionnent selon les prévisions déduites du comportement du modèle ré-. duit. Dans le détail, on remarquera que la forme triangulaire accusée, que les profils avaient à l'origine, est devenue plus trapézoïdale : de légers creusements ont été constatés du côté de la rive droite; ils ont été compensés par des remblais modérés dans le profond thalweg de la rive gauche où il subsiste bien au pied des seuils les fosses mises en évidence par l'étude sur modèle;

UNE ZONE $N^{\circ} 5$ entre l'entrée double et le barrage de retenue. C'est une zone qui se remblaie progressivement pour les crues modérćes en raison du prélèvement du débit dérivé et qui se déblaie aisément lorsque le barrage de retenue est ouvert. Dans cette région, l'effet de courbure continue à se manifester, et il subsiste un chenal profond le long de la rive gauche; mais ce chenal est étroit, la plage de rive droite prenant la forme convexe des lits en état de remblaiement, et le fond est surélevé.

Les cartes à courbes de niveaux des fonds du lit, ou mieux les plans de sondages (dans lesquels les profondeurs sont mesurées par rapport à la retenue du fleuve en étiage), représentent plus particulièrement la situation en juillet 1955 


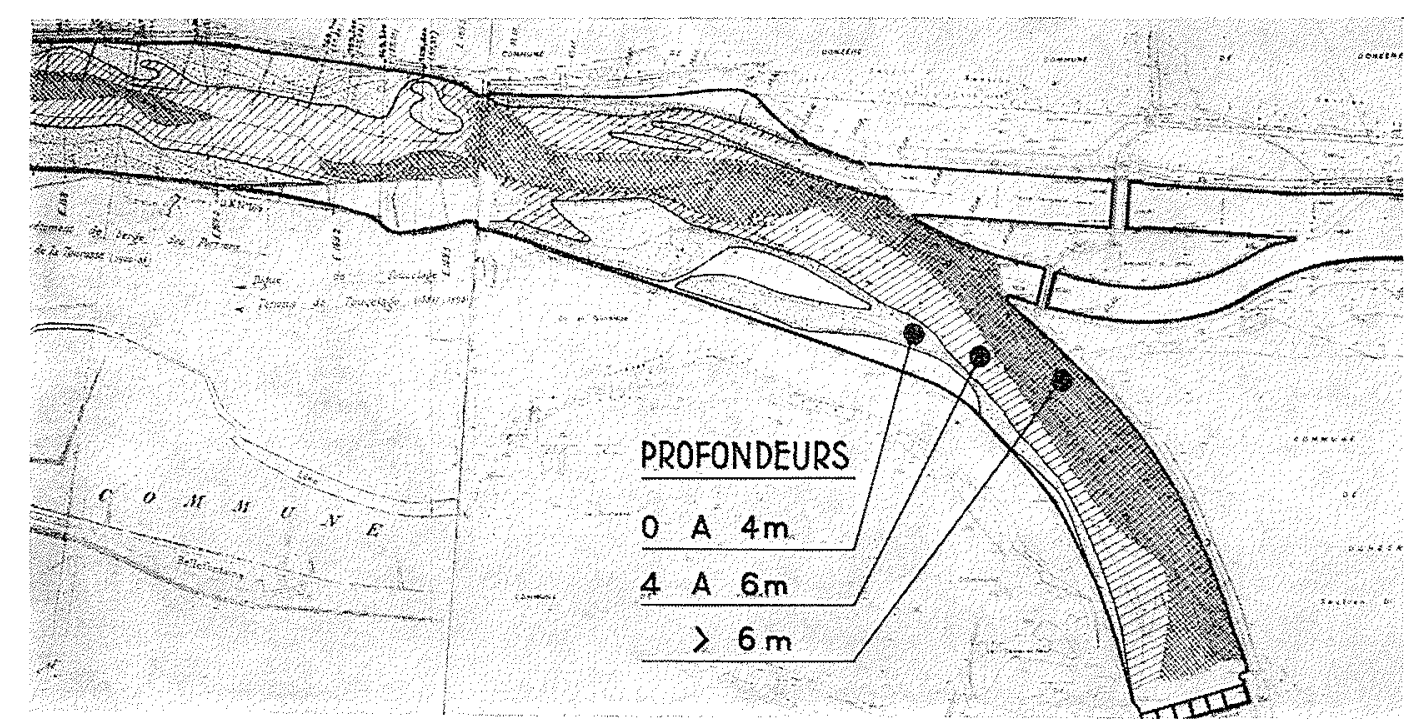

Fis. 8. - Plan de sondage initial au voisinage de la prise (juillet 1952).

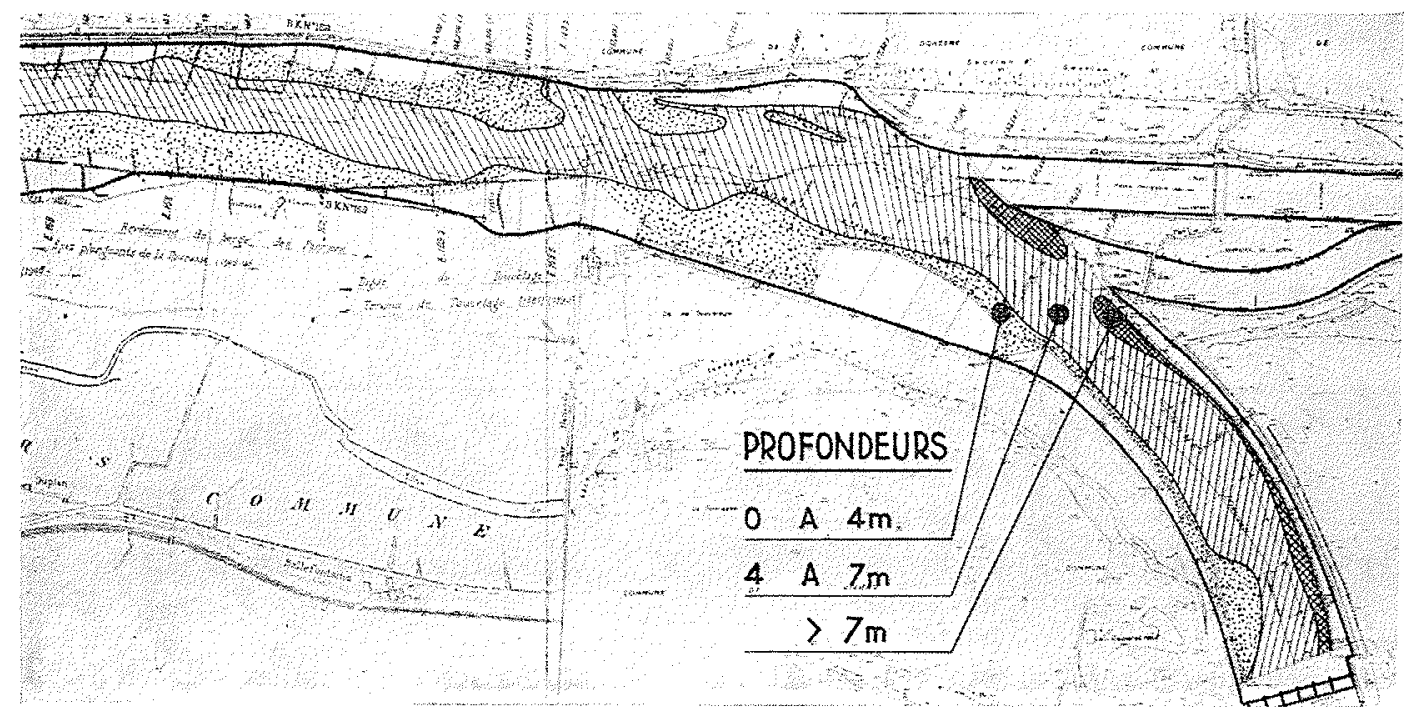

Fig. 9. - Plan de sondage (juillet 1955).

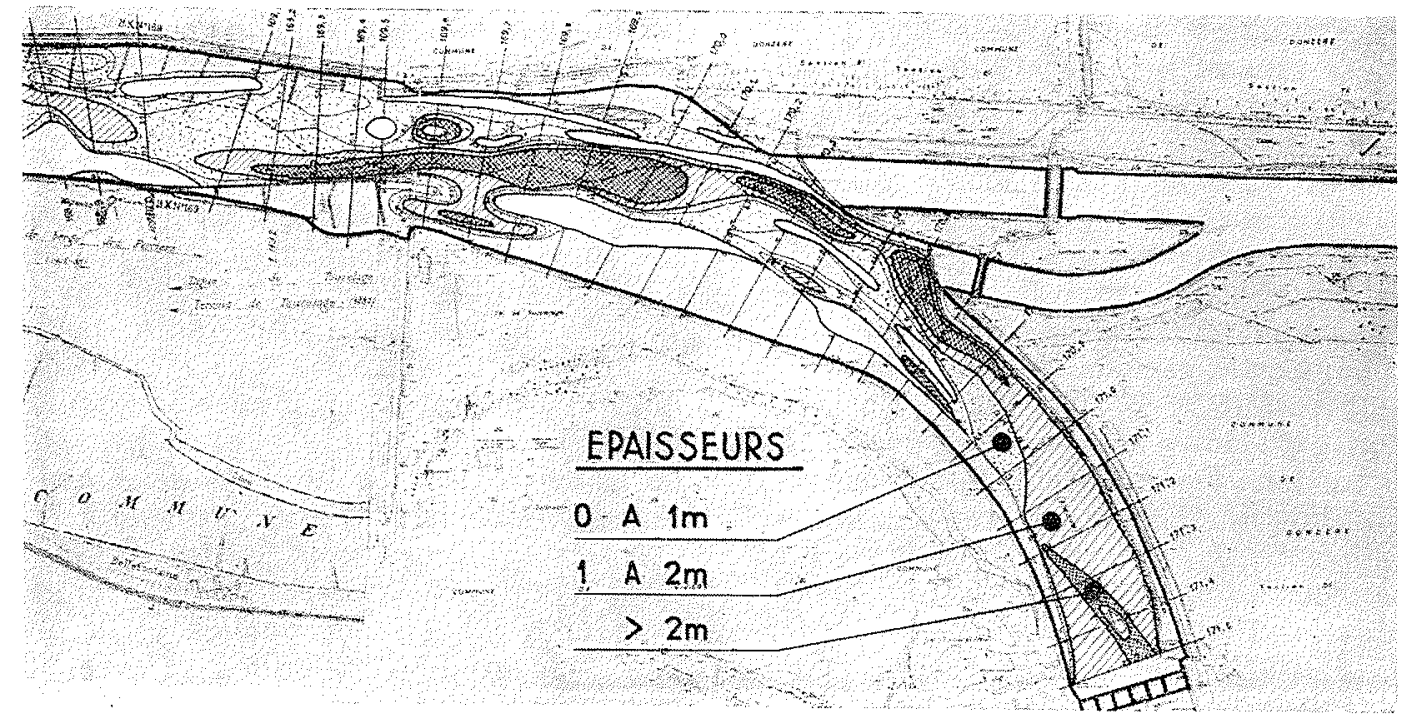

Fra. 10. - Epaisseur des remblais (juillet 1955). 


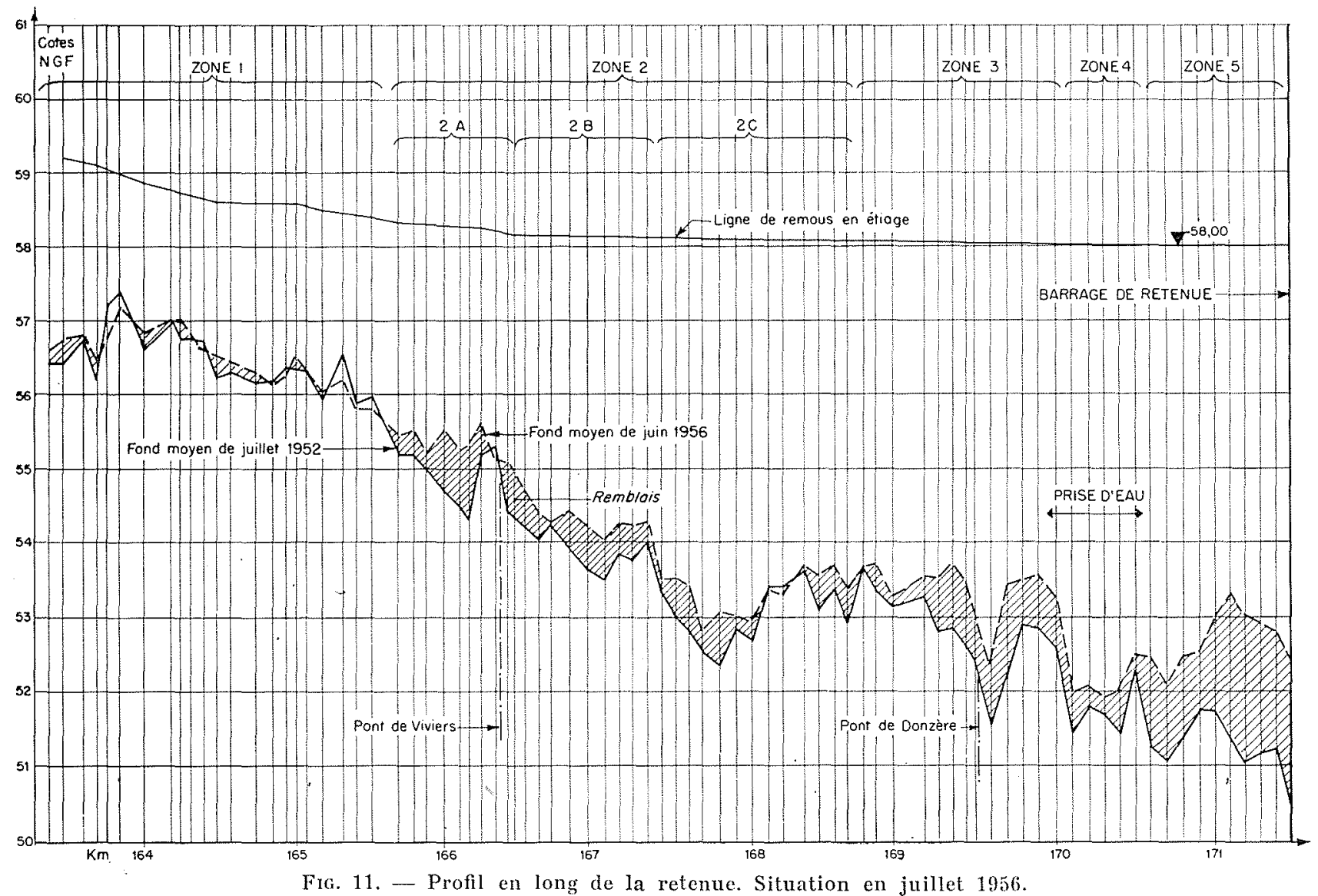

comparativement à celle qui existait au moment de la mise en eau. On y reconnaît les zones que nous venons de décrire (fig. $\mathrm{n}^{\circ} 8,9$ et 10 ).

Nous avons également reporté sur un profil en long de la retenue la situation des déblais et des remblais en juillet 1956 (fig. 11).

D'une façon globale, l'engravement de la retenue a progressé d'une manière assez régulière depuis la mise en eau, jusqu'à atteindre un volume de $500000 \mathrm{~m}^{3}$ lorsqu'est survenue la crue importante de décembre 1954, qui a été immédiatement suivie de deux autres crues très importantes. L'ouverture du barrage et les manœuvres d'exploitation effectuées pendant les trois crues de l'hiver 1954-1955, jointes au fait que, dans leur ensemble, peu de matériaux ont été charriés pendant cette période, ont fait régresser l'engravement jusque vers $300000 \mathrm{~m}^{3}$. Ensuite, l'engravement a de nouveau progressé sous l'effet de crues modérées pour atteindre $800000 \mathrm{~m}^{3}$ en juin 1956. Puis une chasse effectuée au cours de la crue moyenne de septembre 1956 a permis de ramener l'engravement à $600000 \mathrm{~m}^{3}$. Enfin la crue de février 1956 a peu modifié les fonds.

Telle est la situation de la retenue de Donzère au moment de la mise en eau de la retenue de Montélimar:

\section{F. - Evolution des fonds du Rhône court- circuité.}

Le Rhône court-circuité, dont il n'est utile de relever les fonds qu'après de fortes crues nécessitant l'ouverture du barrage, ou après des chasses, a fait l'objet de quatre relevés complets en décembre 1953, en juillet 1955 après les fortes crues de l'hiver 1954-1955, en janvier 1957 et en avril 1957, exclusion faite de relevés partiels dans les zones sensibles ou que l'on craignait telles.

Les constatations faites sont les suivantes:

Depuis la mise en service de l'aménagement de Donzère jusqu'aux fortes crues de l'hiver 1954-1955, les fonds du Rhône court-circuité n'ont pratiquement pas varié. Tout au plus remarque-t-on un léger creusement sur $2 \mathrm{~km}$ de longueur et un faible engravement de l'ordre de $20000 \mathrm{~m}^{3}$ au confluent de l'Ardèche, dû vraisemblablement à une crue de cette rivière.

Après les crues importantes de l'hiver 19541955 ayant nécessité l'ouverture totale du barrage et à la suite desquelles on a observé un dégravement de la retenue, on a constaté, conformément aux prévisions, un remblaiement de $100000 \mathrm{~m}^{3}$ immédiatement à l'aval du barrage 
et étalé sur $1 \mathrm{~km}$, ainsi qu'un remblaiement de $150000 \mathrm{~m}^{3}$ juste en amont de la restitution. Ce dernier remblaiement, provoqué par le remous d'exhaussement dû à l'afflux du débit dérivé, est analogue au remblaiement d'une haute retenue : la plage de galets s'avance en recouvrant indistinctement les mouilles et les seuils.

Par contre, sur les $15 \mathrm{~km}$ medians, il s'est produit un dégravement de $450000 \mathrm{~m}^{3}$. Dans le reste du Rhône court-circuité et notamment dans les $8 \mathrm{~km}$ à l'aval du remblai qui s'est formé près du barrage, les fonds sont restés très stables. Au total, le Rhône court-circuité s'était dégravé de $200000 \mathrm{~m}^{3}$.

Après la crue moyenne de septembre 1956 et la chasse qui a permis de dégraver la retenue (de $200.000 \mathrm{~m}^{3}$ environ), on a constaté que les remblais près de la restitution se sont étalés et partiellement résorbés $\left(70000 \mathrm{~m}^{3}\right.$ contre $110000 \mathrm{~m}^{3}$ ) et que les remblais près du barrage sont restés inchangés $\left(100000 \mathrm{~m}^{3}\right)$. Dans la région médiane, qui s'était précédemment creusée, des remblaiements représentant $350000 \mathrm{~m}^{3}$. ont eu lieu, si bien que les fonds initiaux ont été sensiblement retrouvés.

Enfin, après la crue de février 1957, on a observé que les remblais près de la restitution avaient continué de se résorber, tandis que les remblais près du barrage avaient un peu augmenté (en passant de $100000 \mathrm{~m}^{3}$ à $125000 \mathrm{~m}^{3}$ ).
Au total, et par rapport aux fonds initiaux, le Rhòne court-circuité, après quelques fluctuations, ne s'est remblayé que de $50000 \mathrm{~m}^{3}$, ce qui est insignifiant.

\section{G. - Evolution des fonds à l'aval de la resti- tution.}

Quatre relevés complets des fonds ont été effectués à l'aval de la restitution. En outre, de nombreuses lignes d'eau ont été relevées et des jaugeages ont été effectués.

II résulte de ces observations que le Rhône, à l'aval de la restitution, s'est déblayé de $300000 \mathrm{~m}^{3}$ sur $7 \mathrm{~km}$ de longueur, mais non uniformément. Le déblaiement était de $100000 \mathrm{~m}^{3}$ après les crues de l'hiver 1954-1955. Il a ensuite progressé pour atteindre $250000 \mathrm{~m}^{3}$ en décembre 1955 et $300000 \mathrm{~m}^{3}$ en mars 1957 , les nouveaux creusements se produisant entre la restitution et le seuil de Saint-Etienne-des-Sorts. Il semble que sur ce seuil et à son aval, on aurait atteint une certaine stabilisation, à partir de laquelle les évolutions seraient lentes.

Ces dégravements ont eu une très faible influence sur les lignes d'eau. A la restitution même, l'abaissement de l'étiage paraît être de l'ordre de $10 \mathrm{~cm}$. Par contre, et cela est le plus important pour la navigation, on n'a pas cons-

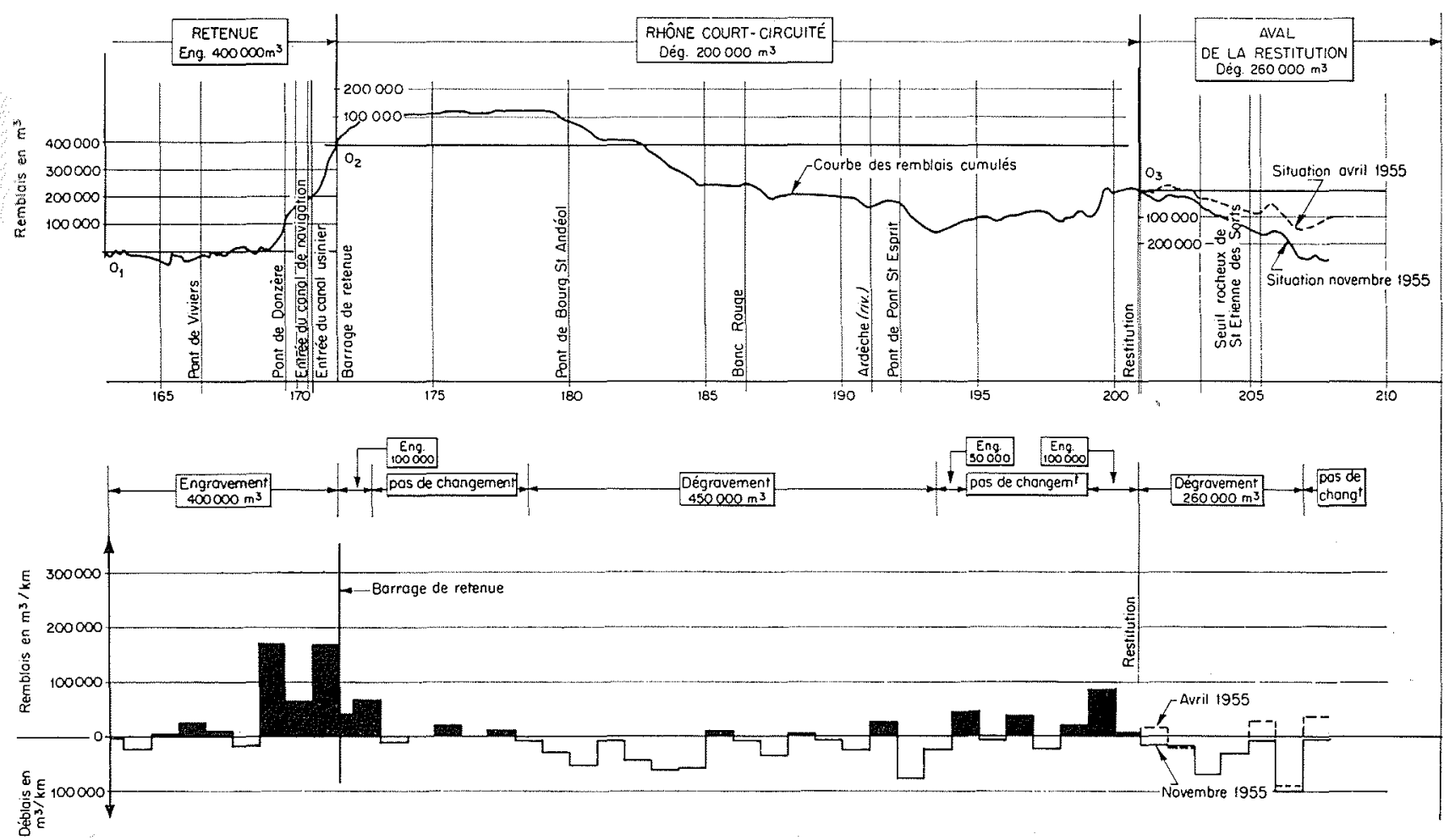

FIG. 12. - Evolution des fonds de l'ensemble de l'aménagement. Situation en juillet 1955. 
taté de modification des lignes d'eau sur le seuil rocheux, ni à son aval.

Pour tout le secteur du Rhône intéressé par l'aménagement, nous avons enfin tracé le graphique des remblais cumulés, qui donne une vue d'ensemble des phénomènes dont nous venons d'exposer le détail (fig. 12).

\section{H. - Charriage du Rhône observé depuis la mise en eau de l'aménagernent de Donzère-Mondragon.}

Comme nous disposons de plusieurs relevés des fonds, qui ont permis de mettre en évidence certains transports de matériaux, il est possible de déterminer le volume de gravier qui a dû pénétrer dans la retenue lors de chaque crue, ainsi que la loi expérimentale de charriage du Rhône, c'est-à-dire la courbe définissant le débit solide en fonction du débìt liquide, pour autant qu'il existe une correspondance biunivoque entre ces deux quantités.

\section{VOLUMES DÉPosés DANS LA RETENUE}

A LA SUITE DE CHACUNE DES CRUES :

Pour chaque intervalle de temps séparant deux relevés consécutifs des fonds de la retenue, on peut déterminer, par les procédés indiqués tout à l'heure, les volumes cumulés ou non des dépôts manifestés dans la retenue (en milliers de $\mathrm{m}^{3}$ ) : volume ( $T$ ) des graviers qui traverse le barrage est égal à la différence des deux volumes suivants :

- les volumes (S) charriés par le Rhône à l'amont de la retenue ou enlevés dans la retenue par déblaiement (D);

- les volumes déposés par remblaiement dans la retenue, qu'ils y soient restés $(R)$, ou qu'ils aient été dragués et mis en dépôt à terre ou dans les casiers (V).

On a donc $\mathrm{T}=\mathrm{S}-\mathrm{D}-(\mathrm{R}+\mathrm{V})$, d'où l'on tire le volume cherché $(S)$ charrié par le Rhône à l'amont de l'aménagement :

$$
\mathrm{S}=\mathrm{R}+\mathrm{V}+\mathrm{T}-\mathrm{D}
$$

Dans la formule précédente, grâce à laquelle nous cherchons à déterminer les matériaux (S) ayant pénétré dans la retenue, nous connaissons, par l'observation des fonds, les remblais (R) et les déblais (D) de la retenue et, d'après le travail de la drague, le volume (V) dragué. Quant au volume $(T)$ ayant transité à travers le barrage, il n'est pas directement accessible et risque d'échapper à l'analyse. Nous sommes conduits à faire deux hypothèses extrêmes :

- L'hypothése minimum, dans laquelle nous ne compterons que le volume de graviers dont le mouvement a pu être décelé directement : déblais observés entre prise et barrage, qui ont par conséquent sûrement franchi celui-ci, dépôts ob-

TABI.EAU II

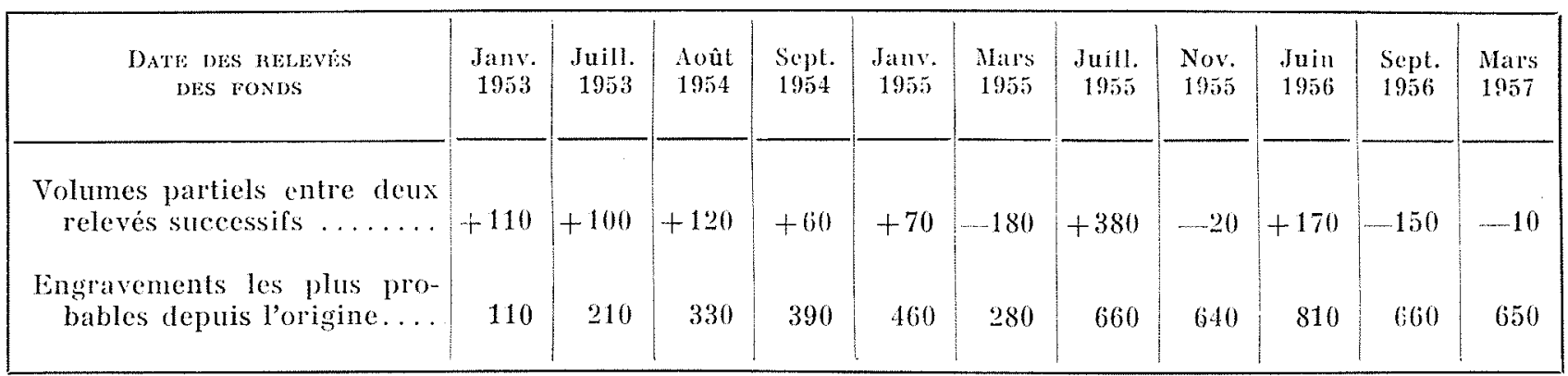

\section{VOLUMES DE GRAVIER}

AYANT PÉNETRE DANS LA RETENUE :

On n'a malheureusement aucun moyen d'évaluer directement le cube de gravier qui a pu pénétrer dans la retenue, mais grâce à un bilan général, on peut mesurer ou apprécier ce qui y est resté el ce qui en est parti, et en déduire pour chaque période considérée le volume des apports solides.

Pour chaque intervalle de temps séparant deux relevés consécutifs des fonds de la retenue, le servés dans le Rhône court-circuité en l'absence de tout dégravement dans cette zone.

Nous admettrons que $T_{m}=\mathrm{R}^{\prime}-\mathrm{D}^{\prime}$ représente les dépôts $R^{\prime}$ observés dans le Rhône courtcircuité ou, lorsqu'il n'y a pas eu de relevés des fonds du Rhône court-circuité en même temps que des fonds de la retenue, les déblais observés entre la prise et le barrage (et qui ont franchi celui-ci).

- L'hypothèse maximum, qui consiste à admettre que le Rhône court-circuité a toujours été saturé en matẻriaux solides. On ajoutera à 
la capacité de transport $G^{\prime}$ de celui-ci, calculée très largement, les remblais $R^{\prime}$ constatés dans ce secteur du fleuve et on en retranchera, s'il y a lieu, le volume des dégravements observés $D^{\prime}$. On a done $\mathrm{T}_{\mathrm{M}}=\mathrm{G}^{\prime}+\mathrm{R}^{\prime}-\mathrm{D}^{\prime}$, étant entendu que, dans l'estimation des remblais, on ne tient compte ni de l'engravement constaté près de la restitution puisqu'il procède d'un autre phénomène, ni des apports éventuels de l'Ardèche qui paraissent bien faibles (de l'ordre de $30000 \mathrm{~m}^{3}$ ).

De la comparaison des équations précédentes :

$$
\begin{aligned}
& \mathrm{T}=\mathrm{S}+(\mathrm{D}-\mathrm{V}-\mathrm{R}) \\
& \mathrm{T}_{\mathrm{M}}=\mathrm{G}^{\prime}+\left(\mathrm{R}^{\prime}-\mathrm{D}^{\prime}\right)
\end{aligned}
$$

il résulte que, si le déblaiement net de la rete- de l'aménagement, seront définis par une fourchette. Cette fourchette n'est d'ailleurs pas très large, car pour toutes les crues ne dépassant pas $3000 \mathrm{~m}^{3} / \mathrm{s}$ (et elles sont relativement fréquentes), le débit du Rhône court-circuité ne dépasse pas $1500 \mathrm{~m}^{3} / \mathrm{s}$, qui semble bien être le seuil du charriage généralisé sur le Rhòne : dans ce cas, les deux hypothèses minimum et maximum se confondent. En l'espace de cinc ans, la marge d'imprécision ne concerne que les matériaux ayant franchi le barrage durant les trois mois de crue notable.

Les calculs conduisent aux charriages probables suivants, pour chaque période séparant deux relevés des fonds de la retenue (tableau III) :

Ainsi, depuis le début de la mise en service

\begin{tabular}{|c|c|c|c|c|}
\hline $\mathrm{N}^{0}$ & $\begin{array}{l}\text { DATES EXTRÊMES } \\
\text { DE LA PÉRIODE }\end{array}$ & $\begin{array}{l}\text { CRUe Caractéristique } \\
\text { DE CETTE PÉMIODE }\end{array}$ & $\begin{array}{l}\text { DÉBIT } \\
\text { MAXMUM } \\
\mathrm{m}^{3} / \mathrm{s}\end{array}$ & $\begin{array}{l}\text { CharRiage Du RhoNe } \\
\text { EN AMONT DE L'AMENAGEMENT } \\
m^{3}\end{array}$ \\
\hline \multirow[t]{2}{*}{$\begin{array}{r}1 \\
2 \\
3 \\
4 \\
5 \\
6 \\
7 \\
8 \\
9 \\
10\end{array}$} & 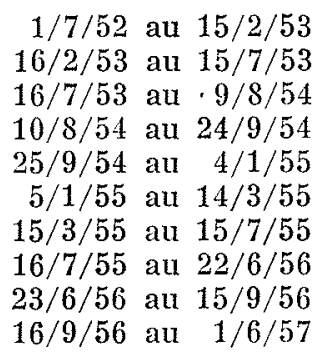 & $\begin{array}{c}\text { hiver } 1952 / 53 \\
\text { juin } 1953 \\
\text { avril } 1954 \\
\text { août } 1954 \\
\text { dècoembre } 1954 \\
\text { janv./fév. } 1955 \\
\text { juin } 1955 \\
\text { janv./avril } 1956 \\
\text { septembre } 1956 \\
\text { février } 1957\end{array}$ & $\begin{array}{l}4.100 \\
2.700 \\
2.500 \\
3.400 \\
5.430 \\
6.100 \\
3.200 \\
2.850 \\
4.600 \\
5.800\end{array}$ & 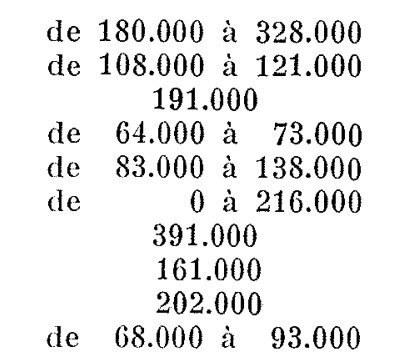 \\
\hline & $1 / 7 / 52$ au $1 / 6 / 57$ & & & $\begin{array}{cc}\text { de } & 1.450 .000 \mathrm{~m}^{3} \\
\dot{\mathrm{a}} & 1.900 .000 \mathrm{~m}^{3}\end{array}$ \\
\hline
\end{tabular}

TABLEAU III

nue ( $\mathrm{D}-\mathrm{V}-\mathrm{R}$ ) excède le remblaiement net du Rhône court-circuité $\left(R^{\prime}-D^{\prime}\right)$, on a forcément :

$$
S<G^{\prime}
$$

autrement dit, le débit solide arrivant en amont de l'aménagement est inférieur à la capacité de transport du Rhône court-circuité.

Cette dernière capacité étant relativement faible en raison du débit dérivé, on voit que, dans le cas considéré, le débit solide arrivant de l'amont est encore inférieur et par conséquent très réduit en regard du débit naturel du Rhône.

Remarquons également que si la retenue s'est déblayée sans qu'il apparaisse dans le Rhône court-circuité des dépôts plus importants que ce déblaiement, le résultat ci-dessus $\mathrm{S}<\mathrm{G}^{\prime}=0$ conduit à admettre un charriage nul pour le Rhône en amont de l'aménagement.

Ainsi, le charriage du Rhône court-circuité, et, par voie de calcul, celui du fleuve en amont de l'aménagement de Donzère, en juillet 1952, jusqu'en juin 1957 , le charriage probable a été compris entre $1450000 \mathrm{~m}^{3}$ et $1900000 \mathrm{~m}^{3}$. L'écart n'est pas très important $( \pm 15 \%$ par rapport à la moyenne) et l'on peut tabler avec une assez grande probabilité d'exactitude sur un charriage d'environ $1700000 \mathrm{~m}^{3}$ pour les cinq premières années d'exploitation, soit en moyenne $350000 \mathrm{~m}^{3}$ par an.

\section{Loi expérimentale de GHarriage du Rhône :}

Ayant déterminé les apports de chaque crue et connaissant par ailleurs pour chacune d'entre elles les débits moyens journaliers, il est possible d'en déduire une loi expérimentale provisoire de charriage du Rhône, c'est-à-dire une correspondance entre le débit solide et le débit liquide telle qu'elle permette de retrouver les apports globaux de chaque crue. 
Les résultats des calculs nous ont conduits à la loi expérimentale suivante (tabl. IV et fig. 13):

\section{TABLEAU IV}

\begin{tabular}{|c|c|}
\hline $\begin{array}{c}\text { DÉBIT LIQUide } \\
Q \mathrm{~m}^{3} / \mathrm{s}\end{array}$ & $\begin{array}{c}\text { Capacité DE TRANSPORT } \\
\text { EXPÉRIMENTALF } \\
\mathrm{G}_{o} \mathrm{~m}^{3} / \text { jour }\end{array}$ \\
\hline & \\
1.500 & 0 \\
2.000 & 3.500 \\
3.000 & 7.000 \\
4.000 & 9.750 \\
5.000 & 11.000 \\
6.000 & 12.000 \\
\hline
\end{tabular}

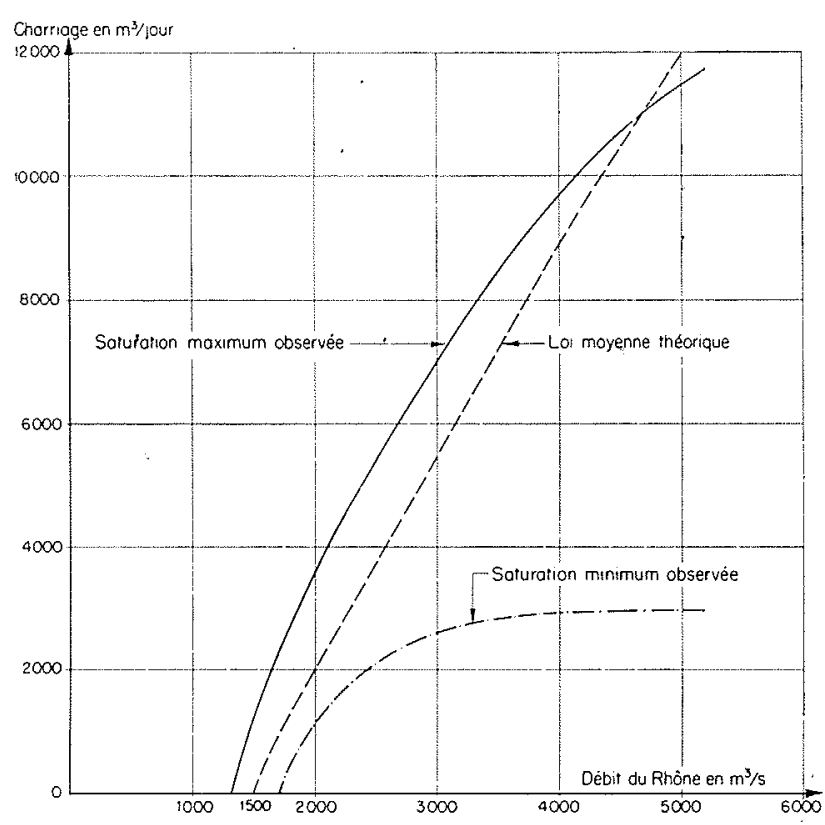

Fic. 13. - Charriage observé sur le Rhòne.

Le débit solide maximum augmente d'abord très rapidement avec le débit liquide, puis il plafonne vraisemblablement à $12000 \mathrm{~m}^{3} / \mathrm{jour}$ environ à partir d'un débit liquide de l'ordre de $6000 \mathrm{~m}^{3} / \mathrm{s}$.

La loi précédente correspond à la saturation maximum du Rhône en débit solide. Au cours d'une crue, le fleuve peut charrier notablement moins de matériaux; théoriquement, le charriage peut même être nul. Les observations faites ont montré que la saturation minimum représentait, pour les forts débits, le quart de la capacité de transport. Tel aura été le cas des fortes crues de l'hiver 1954-1955. Ainsi, pour un même débit liquide, le charriage du Rhône oscille d'une crue à l'autre entre un minimum et un maximum. Le charriage effectif dépend de l'état du lit au mo- ment où se produit la crue, lequel a été influencé par les crues précédentes; il dépend également de la durée de la crue : il semble qu'une crue très rapide n'a pas le temps de mobiliser autant de malériaux qu'elle aurait pu le faire si le débit avait augmenté progressivement.

Il ne semble pas d'autre part y avoir de corrélation entre l'état de saturation ou de non-saturation du fleuve et les bassins versants origines de la crue. Dans le cours moyen ou inférieur, les crues générales du Rhône correspondent en réalité à un « défilé » des crues des divers affluents dans l'ordre suivant: Drôme, Eyrieux, Isère, Ain, Saône. Il est pratiquement impossible d'analyser les apports solides dus à chaque affluent. D'autre part, le débit solide de charriage se déplace de dune en dune, en moyenne beaucoup plus lentement que le débit liquide.

\section{I. - Débit solide théorique du Rhône d'après les lois de charriage (1).}

Il y a quelques années, lorsque la Compagnie Nationale du Rhône a confié au Laboratoire National d'Hydraulique de Chatou l'étude sur modèle réduit de l'entrée de la dérivation de Donzère-Mondragon, ce laboratoire a évalué le débit solide du Rhône d'après les lois théoriques de charriage.

Comme il s'agissait de réaliser le premier grand aménagement du Rhône comportant une dérivation et qu'il était couramment admis que le débit solide du Rhône était très important, toutes précautions avaient été prises pour étudier l'entrée de la dérivation dans les circonstances les plus défavorables. Ainsi le Laboratoire N'ational d'Hydraulique a appliqué la loi de M. le Professeur Meyer-Peter en lui apportant certaines simplifications de façon à trouver des résultats dans le sens de la sécurité. Le Rhône a été assimilé à un canal rectiligne et de section rectangulaire indéfiniment affouillable et il a été admis que tout le débit liquide du Rhône engendrait le charriage, alors que, du fait des débordements, une fraction seulement de ce débit intervient. II a également été admis que la rugosité du lit résultait seulement de la granulométrie des matériaux, sans faire intervenir la rugosité complémentaire de forme du lit provoquée par les ouvrages de régularisation et les variations

(1) Il a été rendu compte de l'estimation théorique du débit solide du Rhône, faite à l'aide des lois de Meyer-Peter dans la communication présentée au Septième Congrès de l'Association Internationale de Recherches Hydrauliques (Lisbonne, juillet 1957) par MM. Gemaehling, Ginocchio et Chabert : « Comparaison entre évaluations théoriques et expérimentales du débit solide dans le Tiers Central du Rhône ». 
de profondeur et de largeur lorsqu'on passe d'un profil à un autre.

C'est à partir de ces évaluations excédentaires, qui conduisent à un charriage moyen annuel du Rhône à Donzère de $1200000 \mathrm{~m}^{3} / \mathrm{an}$, qu'ont été effectués les essais de la prise de Donzère, ainsi que ceux de la prise de Montélimar.

Diverses circonstances, en particulier le comportement des modèles de Montélimar et de Baix pour des essais de longue durée, ont conduit récemment le Laboratoire National d'Hydraulique a reprendre les calculs de base sans leur apporter les simplifications faites. L'expérience acquise par cinq années d'observations des fonds intéressés par l'aménagement de Donzère-Mondragon montrait au surplus que, pour comparer valablement le charriage effectif du Rhône et celui calculé théoriquement, il convenait de ne pas négliger les coefficients modérateurs se trouvant dans la formule utilisée:

Sur ces nouvelles bașes, qui tiennent compte que le Rhône comporte des ouvrages de régularisation et un champ d'inondation, la capacité de transport du Rhône à Donzère est, en année moyenne, de l'ordre de $500000 \mathrm{~m}^{3} / \mathrm{an}$, soit deux fois et demi moins que l'évaluation antérieure. Les capacités de transport pour divers débits liquides sont les suivantes:

\section{TABLEAU $V$}

\begin{tabular}{|c|c|c|}
\hline \multirow{2}{*}{$\begin{array}{l}\text { DÉBTT Liguide } \\
\text { en } \mathrm{m}^{3} / \mathrm{s}\end{array}$} & \multicolumn{2}{|c|}{$\begin{array}{c}\text { CapacitÉ DE TRANSPORT } \\
\text { THEORIOUE }\end{array}$} \\
\hline & en $t /$ jour & en $\mathrm{m}^{3} /$ joul \\
\hline $\begin{array}{l}1.500 \\
2.000 \\
3.000 \\
4.000 \\
5.000 \\
6.000\end{array}$ & $\begin{array}{r}0 \\
3.500 \\
10.000 \\
16.200 \\
22.000 \\
27.000\end{array}$ & $\begin{array}{r}0 \\
2.000 \\
5.500 \\
9.000 \\
12.200 \\
15.000\end{array}$ \\
\hline
\end{tabular}

Ơn voit que la capacité de transport croît suivant une loi parabolique avec un exposant inférieur à l'unité, cette croissance étant beaucoup moins rapide que suivant la loi précédente.

Il apparaît ainsi la très grande différence existant d'une part entre la capacité de transport du Rhône, fleuve dont le lit est fixé par des ouvrages de régularisation et qui a des débits de débordement importants, et d'autre part la capacité de transport d'un canal ayant une pente uniforme, celle moyenne du Rhône, une largeur constante, celle du lit mineur du Rhòne, et un fond très affouillable, sans épis ni rocher, fond qui serait uniquement constitué sur une très grande profondeur par des matériaux ayant la même granulométrie que celle du lit du Rhône (fig. 14).

Les évaluations de la capacité de transport du Rhône qui viennent d'être citées sont des moyennes qui résultent de calculs effectués sur une vingtaine de profils choisis sur un tronçon de Rhốne de $3 \mathrm{~km}$ de longueur. Dans le détail, on trouve des valeurs assez dispersées. Pour certaines sections, le charriage ne commencerait qu'à partir de $2000 \mathrm{~m}^{3} / \mathrm{s}$; pour d'autres, ce serait dès $1200 \mathrm{~m}^{3} / \mathrm{s}$. Il a été admis que le charriage débutait en moyenne pour le débit intermédiaire de $1500 \mathrm{~m}^{3} / \mathrm{s}$, chiffre résultant d'une mesure faite à l'hydrophone, il $y^{\prime}$ a quelques années, lors des études de l'entrée de Donzère.

L'expérience confirme assez bien ces déductions théoriques. Le charriage n'apparaît pas si-
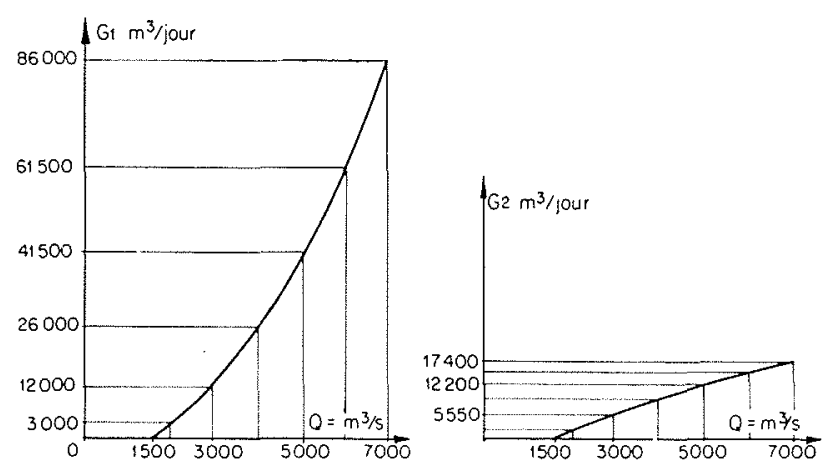

Frg. 14. - Capaciés de transport théoriques d'un canal au fond aflouillable $(\dot{a} g$.$) et du Rhône (\dot{a} d r$.$) .$

multanément sur toute la surface du lit, et ne s'arrête pas simultanément à la décrue:

- lorsque nos travaux de barrages provoquent une obstruction temporaire, et par conséquent une augmentation locale de la puissance de transport du fleuve, on constate que les déblais enlevés dans la section rétrécie vont se déposer sur le seuil situé en aval : la capacité de transport du fleuve est donc plus forte dans les mouilles que sur les seuils;

- à la fin d'une grande crue, on trouve couramment les seuils surélevés de l'ordre d'un mètre (alors que les mouilles sont restées à leur profil antérieur), et ensuite cette surélévation disparait en un ou deux mois pour des débits de l'ordre de 1500 à $2000 \mathrm{~m}^{3} / \mathrm{s}$;

- au total, il est permis de penser que les galets vont souvent d'un seuil au seuil suivant, sans s'arrêter dans la mouille intermédiaire. 


\section{J. - Comparaison entre le charriage observé et le charriage calculé par les lois théoriques.}

Une première comparaison entre le charriage observé et le charriage calculé par des lois théoriques peut être faite globalement en prenant en considération les débits qui se sont écoulés dans la retenue depuis la mise en eau de l'aménagement de Donzère jusqu'au dernier relevé des fonds, sans faire intervenir les relevés intermédiaires. On calcule ainsi la capacité de transport $\mathrm{G}$ du Rhône à l'amont de l'aménagement, ce qui représente le débit solide maximum théorique ayant pu arriver dans la retenue dans le cas de saturation permanente du Rhône. On trouve $\mathrm{G}=1800000 \mathrm{~m}^{3}$.

Or, nous avons vu que le charriage observé était de $1700000 \mathrm{~m}^{3}$. Il y a une excellente concordance entre la théorie et les faits pour les résultats globaux.

Une deuxième méthode de comparaison consiste à faire intervenir non seulement la capacité théorique de transport $G$ du Rhòne à l'amont de l'aménagement, soit $1800000 \mathrm{~m}^{3}$, mais également la capacité théorique de transport $G^{\prime}$ du Rhône court-circuité, qui représente le débit solide maximum pouvant arriver à la restitution dans la même hypothèse, soit $\mathrm{G}^{\prime}=500000^{\prime} \mathrm{m}^{3}$. La différence entre ces deux capacités théoriques de transport: $\mathrm{G}-\mathrm{G}^{\prime}=1300000 \mathrm{~m}^{3}$, représente le débit solide maximum qui a pu se déposer dans la retenue et à l'aval du barrage, si le Rhône a toujours été saturé.

Or, le dépôt final qui s'est effectivement formé dans la retenue $\left(650000 \mathrm{~m}^{3}\right)$ et à l'aval du barrage $\left(125000 \mathrm{~m}^{3}\right)$ majoré de la partie du volume des matériaux dragués qui ne sont plus mobilisables $\left(100000 \mathrm{~m}^{3}\right)$ pour avoir été mis en dépôt à terre ou pour avoir été déversés dans des casiers du Rhône, représente un volume global de $875000 \mathrm{~m}^{3}$, soit les deux tiers du volume théorique. Cela signifie que, dans le détail, il existe des différences entre la loi théorique de charriage el la loi réelle. En procédant au calcul crue par crue, il est possible de comparer les volumes résultant du calcul aux volumes réels et d'essayer d'expliquer les différences (tableau VI).

L'étude théorique s'applique à des graviers correspondant à une granulométrie constante; elle donnerait des tonnages bien plus importants i l'on admettait que les matériaux sont plus fins pour les faibles débits. C'est ce que confirme l'expérience : on a observé, lors de la crue de juin $1955\left(3200 \mathrm{~m}^{3} / \mathrm{s}\right)$, que les apports, relativement importants, étaient composés de matériaux plus fins que ceux correspondant à la granulométrie générale des alluvions du Rhône.

On peut donc penser que chaque crue, petite ou moyenne, nettoie le lit des matériaux relativement fins apportés par les affluents ou produits par l'usure des gros galets depuis la crue précédente.

Inversement, lorsque de grandes crues se succèdent à peu d'intervalle, la seconde crue trouve un lit nettoyé et pavé de gros éléments, de sorte que son débit solide est très faible : c'est ce qui est arrivé lors des crues de janvier-février 1955, qui succédaient à celle de décembre 1954, et dont les apports ont été insignifiants.

En moyenne, les écarts entre la loi théorique du charriage et la loi expérimentale sont minimes (valeurs vraisemblablement insuffisantes pour les petites crues et excédentaires pour les

TABLEAU VI

\begin{tabular}{|c|c|c|c|}
\hline \multirow[b]{2}{*}{$\mathrm{N}^{\circ}$} & \multirow[b]{2}{*}{ DATE DE LA CRUE } & \multicolumn{2}{|c|}{ Ghakriage (en milliers de $\mathrm{m}^{3}$ ) } \\
\hline & & effectif & theorique \\
\hline 1 & Hiver $1952 / 1953 \ldots \ldots \ldots$ & 180 à 328 & 350 \\
\hline 2 & Juin $1953 \ldots \ldots \ldots \ldots$ & 108 à 121 & 110 \\
\hline 3 & Avril $1954 \ldots \ldots \ldots \ldots$ & 191 & 99 \\
\hline 4 & Août $1954 \ldots \ldots \ldots$ & 64 à 73 & 55 \\
\hline 5 & Décembre $1954 \ldots$ & 83 à 138 & 178 \\
\hline 6 & Janvier-février $1955 \ldots$ & 0 à 216 & 385 \\
\hline 7 & Juin $1955 \ldots \ldots \ldots$ & 391 & 185 \\
\hline 8 & Août $1956 \ldots \ldots \ldots$ & 161 & 145 \\
\hline 9 & Septembre $1956 \ldots$ & 202 & 158 \\
\hline \multirow[t]{2}{*}{10} & Fevrier $1957 \ldots \ldots$ & 68 a 93 & 190 \\
\hline & Charriages globaux ......... & 1.450 à 1.900 & 1.855 \\
\hline
\end{tabular}


(randes). De plus, rappelons que le fleuve, dont lo lit a été bien stabilisé par les ouvrages de régularisation, ne peut pas toujours se saturer on matériaux (tableau VII).
zère-Mondragon, au sujet de la possibilité d'assurer dans de bonnes conditions le maintien du lit et l'exploitation des ouvrages malgré les apports solides du fleuve.

TABLEAU VII

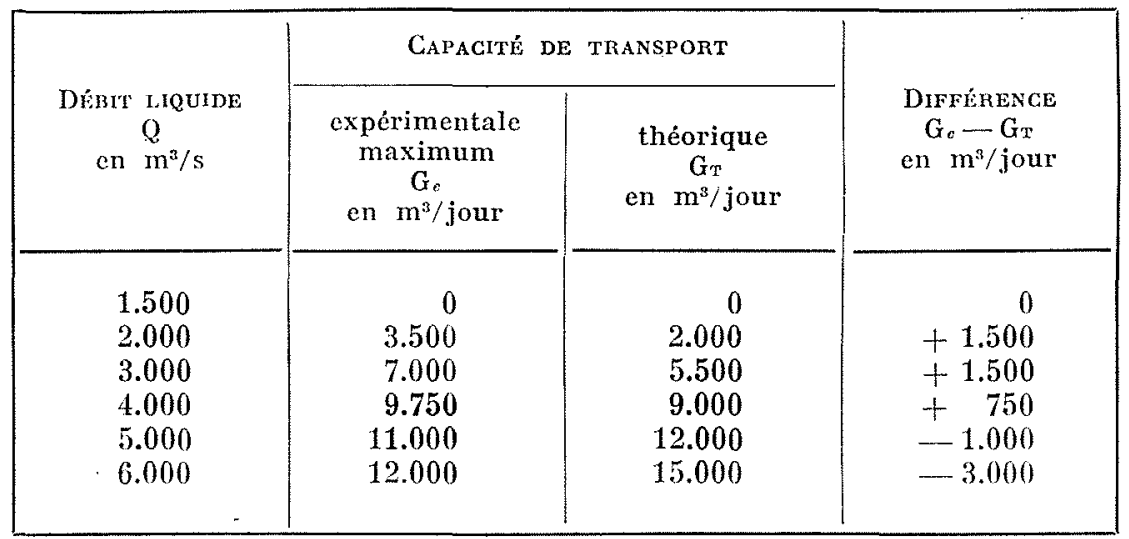

\section{K. - Matériaux en suspension.}

Dans tout ce qui précède, nous avons délibérément limité notre propos au débit solide de charriage et' nous n'avons 'pas parlé du débit solide en suspension dans les eaux du Rhône. Nous nous contenterons de donner ici quelques indications très brèves sur ce sujet, qui pourrait laire l'objet d'une étude spéciale. Les eaux sont chargées en permanence d'une vase très fine qui n'a pas tendance à se déposer dans le lit mineur du fleuve en raison de la vitesse du courant. En période de crue, la concentration est beaucoup plus forte, et comme la vitesse de l'eau est souvent faible dans les zones de débordement de son lit majeur, le Rhône laisse déposer des limons qui sont les bienvenus pour l'agriculture.

La teneur des eaux dérivées à Donzère peut atteindre, en eaux moyennes du Rhône, de 1 à 1,5 décigramme par litre, si bien que le canal Iransporte, avec ses $1500 \mathrm{~m}^{3} / \mathrm{s}$, environ 10000 a 15000 tonnes par jour de matières en suspension.

Fort heureusement, ces matières ne se déposent pas en masse dans nos ouvrages, car la vitesse est en général trop élevée pour qu'il y ait dépôt. Il y a cependant une exception au voisinage des écluses où les eaux sont pratiquement immobiles. Malgré les précautions prises, i] se forme quelques dépôts de vase qu'il convient de draguer périodiquement.

\section{L. - Conclusion générale.}

Nous avons évoqué les craintes qu'on avait pu avoir lors des études de l'aménagement de Don-
L'expérience montre qu'après cinq années d'exploitation, rien de tel ne s'est produit :

- il existe bien dans la retenue un certain remblai (qui est de l'ordre de $600000 \mathrm{~m}^{3}$ ) mais le volume de ce remblai ne forme que quelque $7 \%$ du volume de la retenue ( 8 millions de $\mathrm{m}^{3}$ ). Au surplus, les dépôts sont concentrés dans la région profonde de la retenue et n'apportent aucune perturbation sensible à l'écoulement des eaux. La Compagnie a fait construire une drague pour l'entretien de la retenue et du lit, mais il arrive que cet engin, susceptible de draguer à $7 \mathrm{~m}$ de profondeur, ne puisse être utilisé faute de remblais situés à moins de $7 \mathrm{~m}$;

- au droit de la prise d'eau, la section, qui était triangulaire, tend à devenir trapézoïdale, par creusement sur la rive droite et remblaiement du côté de la prise, mais ce remblaiement est très modéré; il laisse le long de la prise un chenal profond où règne un tourbillon à axe horizontal qui maintient de grandes profondeurs;

- en aval du barrage, l'engravement est très modeste (il ne dépasse pas $125000 \mathrm{~m}^{3}$ ); il n'agit pas sur l'écoulement des eaux, et jusqu'à présent nous n'avons pas eu à nous préoccuper de le draguer;

- plus en aval, le lit du Rhône court-circuité, loin de s'exhausser, s'est d'abord creusé et paraît tendre à reprendre son profil initial;

- des dépôts se sont produits en amont de la restitution; ils n'ont pas eu d'action sen- 
sible sur l'écoulement des eaux de crue et ils se sont réduits;

- enfin, en aval de la restitution, l'abaissement du lit est très lent : jusqu'à présent, la ligne d'eau d'étiage ne s'est enfoncée que de $10 \mathrm{~cm}$ à la restitution même et non sur le seuil rocheux de Saint-Etienne-desSorts.

Ces heureux résultats doivent être attribués en premier lieu à la fixation du lit réalisée par l'aménagement à courant libre: grâce à cette fixation, les débits solides restent modérés et sont très loin d'atteindre, pour les fortes crues, les débits solides d'un canal au fond très affouillable.

La surveillance constante de l'évolution des fonds du lit du fleuve au droit de l'aménagement nous a permis de compléter nos connaissances sur le charriage du Rhône et de dégager quel- ques principes essentiels relatifs à l'utilisation des chasses (baisse du niveau de la retenue et diminution du débit dérivé) pour le maintien du lit au voisinage de la retenue. Il est cependant probable que des dragages seront nécessaires de temps en temps dans le Rhône court-circuité, en aval du barrage et en amont de la restitution.

Cette conclusion optimiste a orienté la conception des quatre chutes situées en amont: alors qu'à Donzère le niveau de la retenue au droit de la prise d'eau correspond au niveau naturel pour le débit de la crue annuelle, les niveaux analogues pour les quatre chutes supérieures dépassent celui de la crue millénaire.

Cette situation posera de nouveaux problèmes dont nous pensons pouvoir aborder prochainement l'étude expérimentale, puisque la première de ces chutes, celle de Montélimar, a été mise en service au cours de l'été 1957 alors que, plus en amont, démarraient les travaux de l'aménagement de Baix-Le Logis Neuf.

\section{I S C US I O N}

président: M. DE Rouvmex

M. le Président indique que cette communication a déjà été présentée à la Section d'Hydraulique Fluviale et Maritime le 28 février 1957, par M. Mare Henny, et remercie les conférenciers d'avoir bien voulu résumer à nouveau devant le Comité Technique leurs travaux très remarquables.

M. Fauconnier demande si les cubatures qui ont été faites pour évaluer le charriage annuel du Rhône n'ont pas été entachées par l'omission du cube pouvant avoir transité pendant les fortes crues de l'amont à l'aval du troncon du Rhône ayant fait l'objet des mesures.

M. Gemaehlivg répond en rappelant que le volume ainsi omis ne peut être compté avec précision, mais que l'on peut l'estimer inférieur à la marge d'incertitude comprise entre $1.500 .000 \mathrm{~m}^{3}$ et $1.900 .000^{3}$, soit environ $400.000 \mathrm{~m}^{3}$ en l'espace de cinq années; en effet, la majeure partie du charriage se produit pour des débits compris entre 1.500 et $3.000 \mathrm{~m}^{3} / \mathrm{s}$ et, comme le canal dérive en permanence $1.500 \mathrm{~m}^{3} / \mathrm{s}$, le Rhône court-circuité ne peut charrier de manière systématique un débit solide que pour des crues notables du Rhône qui représentent moins de trois mois sur la période de cinq ans étudiée.

En réponse à une question de M. le Président, M. GEMAEHLING indique que la densité des matériaux ne varie guère : la proportion d'éléments calcaires et siliceux reste à peu près la même et les vides semblent relativement identiques; il y a, cependant, en fait, des crues qui apportent des matériaux plus fins en quantité plus grande.

M. BLANCHET exprime son opinion comme suit :

Les valeurs de débits solides et dépôts dans la zone de Donzère, considérées seulement en valeur relative, semblent confirmer les prévisions exposées au début de la communication et auxquelles on s'attend en général. La retenue amont s'engrave la première et tend rapidement vers un premier équilibre; la branche courtcircuitée commence souvent par se creuser, car elle est privée de matériaux, puis s'engrave en commençant par les zones désavantagées, c'est-à-dire l'aval du barrage et la zone de jonction. Enfin, après la jonction, il y a affouillement. Ce premier stade semble atteint à Donzère Si cette chute restait la seule à être aménagée, la deuxième phase pourrait alors se développer, à savoir l'engraissement du bras court-circuité avec influence vers l'amont dans la retenue, qui reprendrait son engraissement. Le tout s'effectuerait évidemment à une allure très lente en raison de la surface disponible et malgré les déficits encore relativement importants dans l'acheminement des matériaux. Le développement des chutes amont, en harmonisant les apports avec ceux dont est actuellement capable Donzère, peut donc faire arrêter là l'ẻvolution.

M. Gemaenting indique que les observations ont confirmé les prévisions; cependant, l'ordre de grandeur des volumes mesurés au cours des cinq années d'observation est de $350.000 \mathrm{~m}^{3}$ par an, en moyenne, alors que les prévisions étaient de 1 million à 1 million et demi. D'autre part, en faisant abstraction de l'aménagement en amont, un moyen consisterait it recalibrer le lit mineur du Rhône court-circuité de manière à lui permettre, malgré un débit plus faible, d'éliminer davantage de matériaux.

M. Henry indique un autre moyen de défense : lorsque l'engravement du Rhône court-circuité est devenu notable, on peut décider qu'à la crue suivante on diminuera le débit dérivé, ce qui permet de revenir vers l'état naturel.

M. Fauconnier observe que le calcul théorique a conduit, pour la Durance comme pour le Rhône, à surestimer le charriage annuel. Ce n'est qu'à la suite d'essais sur modèle et de recoupements faits en Durance même et sur le Verdon, que l'on a eu la bonne surprise de devoir réduire cette évaluation de un million de metres cubes à $130.000 \mathrm{~m}^{3}$, ce qui a évidemment réduit l'acuité des problèmes qui se posaient à l'E.D.F. pour l'aménagement de la riviere. 